\title{
Proximity interactome of LC3B in normal growth conditions
}

Marie Nollet ${ }^{1}$, Alexander Agrotis ${ }^{2,5^{*}}$, Fanourios Michailidis ${ }^{1,6^{*}}$, Arran David Dokal ${ }^{3}$, Vinothini Rajeeve $^{3}$, Jemima Burden ${ }^{2}$, Thomas D. Nightingale ${ }^{4}$, Pedro Cutillas $^{3}$, Robin Ketteler ${ }^{2}$, and Stéphanie Kermorgant ${ }^{1 \#}$

${ }^{1}$ Spatial Signalling Team, Barts Cancer Institute, Queen Mary University of London, John Vane Science Centre, Charterhouse Square, London EC1M 6BQ, United Kingdom.

${ }^{2}$ MRC Laboratory for Molecular Cell Biology, University College London, London WC1E 6BT, United Kingdom.

${ }^{3}$ Cell Signalling \& Proteomics Group, Barts Cancer Institute, Queen Mary University of London, EC1M 6BQ London, United Kingdom.

${ }^{4}$ Centre for Microvascular Research, William Harvey Research Institute, Barts and the London School of Medicine and Dentistry, Queen Mary University of London, EC1M 6BQ London.

${ }^{5}$ Present address: MRC Protein Phosphorylation \& Ubiquitylation Unit, School of Life Sciences, University of Dundee, DD1 5EH Dundee, United Kingdom.

${ }^{6}$ Present address: The Francis Crick Institute: 1 Midland Rd, NW1 1AT London

* These authors contributed equally to this work

\# Correspondence and requests for materials should be addressed to Stephanie Kermorgant. Email: s.kermorgant@gmul.ac.uk

Keywords: autophagy, APEX2, LC3B, proximity proteomics 


\section{Abstract}

LC3 (Light Chain 3) is a key player of autophagy, a major stress-responsive proteolysis pathway promoting cellular homeostasis. It coordinates the formation and maturation of autophagosomes and recruits cargo to be further degraded upon autophagosome-lysosome fusion. To orchestrate its functions, LC3 binds to multiple proteins from the autophagosomes' inner and outer membranes, but the full extent of these interactions is not known. Moreover, LC3 has been increasingly reported in other cellular locations than the autophagosome, with cellular outcome not fully understood and not all related to autophagy. Furthermore, novel functions of LC3 as well as autophagy can occur in cells growing in a normal medium thus in non-stressed conditions. A better knowledge of the molecule in proximity to LC3 in normal growth conditions will improve the understanding of LC3 function in autophagy and in other cell biology function. Using an APEX2 based proteomic approach, we have detected 407 proteins in proximity to the well-characterised LC3B isoform in non-stress conditions. These include known and novel LC3B proximity proteins, associated with various cell localisation and biological functions. Sixty-nine of these proteins contain a putative LIR (LC3 Interacting Region) including 41 not reported associated to autophagy. Several APEX2 hits were validated by co-immunoprecipitation and co-immunofluorescence. This study uncovers the LC3B global interactome and reveals novel LC3B interactors, irrespective of LC3B localisation and function. This knowledge could be exploited to better understand the role of LC3B in autophagy and non-autophagy cellular processes. 


\section{Introduction}

LC3B (Light Chain 3B), or MAP1LC3B (Microtubule-Associated Proteins 1A/1B light chain 3B), is the best-studied member of the seven protein-family LC3/GABARAP, and a homologue of yeast ATG8 (Autophagy Related 8) (1). The three isoforms LC3 A, B and C (hereafter LC3 when reporting on general functions or according to cited literature), are $17 \mathrm{kDa}$ proteins which localise on autophagosomes, the double membrane vesicles involved in the execution of macro-autophagy (hereafter, autophagy), a major intracellular proteolysis pathway (2). Autophagosome-lysosome fusion leads to the degradation of cytoplasmic material sequestered in the autophagosomes (3), releasing amino-acids for recycling and re-use by the cells (4). Autophagy is induced in response to stress, such as during infection by a pathogen, deprivation of nutrients or hypoxia, thereby promoting cell survival. However, if stress persists autophagy can lead to cell death. Cells can also display basal autophagy, allowing the clearance of long-lived proteins, damaged organelles and protein aggregates (5, 6). Autophagy is often upregulated in cancer cells $(7,8)$ and has been proposed to play a dual role in cancer: tumour suppressor in the early stages of tumorigenesis but pro-survival in advanced tumours (9).

LC3 is required for autophagosome formation and has been considered an autophagosome marker $(10,11)$. Autophagosome biogenesis $(12)$ begins on the isolation membrane, or phagophore, with the assembly of a pre-initiation complex consisting of ULK1, FIP200, ATG101 and ATG13, which leads to activation of the VPS34/BECLIN1 PI3K Class III complex. Subsequent production of $\mathrm{PI}(3) \mathrm{P}$ on the phagophore and binding of proteins such as WIPI2B leads to the recruitment of ATG16L1, which complexes with ATG5 and ATG12, forming a scaffold to attach LC3 to the forming autophagosome (13). For this to occur, LC3 is cleaved by ATG4B to generate LC3-I (cytosolic), capable of binding to ATG7, followed by transfer to ATG3. Finally, the ATG5-ATG12-ATG16L1 complex allows the covalent conjugation of LC3-I to phosphatidylethanolamine (PE), leading to LC3 lipidation, or the conversion of LC3-I to LC3-II $(14,15)$, which is required for autophagosome elongation and closure $(16,17)$. 
LC3 is localised to the inner and outer membranes of the forming and mature autophagosome where it interacts with many proteins. This is often through the presence of a hydrophobic LC3 interacting region (LIR) (18-20). From the inner membrane, LC3 selectively recruits autophagic cargo into the autophagosome, binding the LIR of cargo receptors such as p62/SQSTM $(21,22)$, called selective autophagy. LC3 on the outer membrane of the autophagosome interacts with LIR-containing core autophagy machinery proteins such as ATG1/ULK1 (23), or proteins from the endolysosomal trafficking pathway such as FYVE and coiled-coil-domain-containing protein 1 (FYCO1) (21). This allows autophagosomes to traffic along microtubules and fuse with endocytic vesicles (autophagosome maturation), leading to the formation of degradative autolysosomes and autophagy $(24,25)$.

Non-conventional and non-autophagic functions of LC3 have also been increasingly reported (26). These can alter processes associated with membrane biology including the regulation of ER function $(27,28)$, secretion and exocytosis $(29-34)$ and the non-canonical autophagy pathway LC3-Associated Phagocytosis (LAP) (35), which enables pathogen entry. LC3-positive vesicles may also play the role of signalling platforms (36-39). Non-autophagic functions of LC3 which do not appear related to membrane biology include the regulation of viral or bacterial replication $(40,41)$ and release $(42)$ and pathogen control $(43)$. The soluble form of LC3, LC3-I, is part of slowly diffusing high molecular weight complexes localised in the cytoplasm and the nucleus (44-46) but their function is poorly understood. Furthermore, LC3 was recently shown to conjugate to proteins ("LC3-ylation") akin to ubiquitination (47), although only few candidate LC3-ylated proteins have been identified to date.

Despite the profound involvement of LC3 in cellular processes, autophagy-related or not, many of them remain poorly characterised. Consistent with this, the full repertoire of proteins interacting with LC3 is not known. Here we systematically defined the LC3B interactome by performing an enzymatic proximity tagging approach using an engineered ascorbate peroxidase, APEX2 $(48,49)$. We used HeLa cells stably transfected with an APEX2- and GFPtagged LC3B construct (or the control GFP-LC3B) and cultured them in normal growth conditions. Our study has confirmed many proteins previously reported to be partners of LC3B 
bioRxiv preprint doi: https://doi.org/10.1101/2021.10.08.463639; this version posted October 9, 2021. The copyright holder for this preprint (which was not certified by peer review) is the author/funder. All rights reserved. No reuse allowed without permission.

Nollet et al, 5

and/or present in autophagosomes, including proteins with putative LIR. It has further revealed novel LC3B proximal proteins such as GAPD1 and elF2D. 


\section{Results}

\section{APEX2-GFP-LC3B labels autophagosomes in HeLa cells}

To determine the proteins adjacent to LC3B, we used an APEX2 (Ascorbate Peroxidase 2) proximity labelling approach, originally developed by Rhee et al. (50). We generated HeLa cells stably expressing the APEX2-GFP-LC3B or GFP-LC3B construct (Fig. 1A) as shown by western blot (Fig. 1B). The localisation of APEX2-GFP-LC3B was investigated by confocal microscopy. Several LC3B puncta were easily detected in cells without any treatment (Fig. 1C top panel). Their number was greatly increased upon a three hour treatment with the V-ATPase inhibitor Bafilomycin A1, which impairs lysosomal acidification, leading to an accumulation of autophagosomes (51). LC3B puncta were slightly increased upon treatment with Torin 1, which inhibits mTOR activity, confirming that the APEX2-GFP-LC3B construct allows an increased formation of autophagosomes and an intact autophagic flux (52). Treatment with both drugs led to an increase of LC3B-positive puncta and clear colocalisation with the late endosome/lysosomal marker LAMP1, indicating an autophagosome accumulation (Fig. 1C).

In the presence of $\mathrm{H}_{2} \mathrm{O}_{2}$, APEX2 peroxidase tags triggers the polymerization of the peroxidase substrate, 3,3'-diaminobenzidine (DAB), into a localised precipitate that gives transmission electron microscopy (TEM) contrast after treatment with $\mathrm{OsO}_{4}$. This allows imaging of cellular compartments, with a very good preservation of ultrastructure and a tighter localisation of the TEM stain than with horseradish peroxidase (HRP) (53). Such staining was observed in HeLa APEX2-GFP-LC3B cells following these treatments. Higher magnification electron micrographs revealed that structures positive for APEX2-GFP-LC3B share features of autophagosomes, including double membranes and engulfed cytoplasmic content (Fig. 1D).

These results confirm that APEX2-GFP-LC3B specifically labels autophagosomes. Such an approach would likely be useful to improve the ultrastructural imaging of autophagosomes and could be used for proximity proteome profiling. 


\section{APEX2 workflow and validation of the method}

Fig. 2A illustrates the workflow of our study aimed at determining the LC3B proximity proteome. HeLa APEX2-GFP-LC3B and HeLa GFP-LC3B cells, cultured for 24 hours in full medium (containing 10\% FBS), were treated with biotin phenol for 30 minutes and $\mathrm{H}_{2} \mathrm{O}_{2}$ for 1 minute, allowing APEX2 to generate biotin-phenoxyl radicals that covalently tag proximal endogenous proteins within $20 \mathrm{~nm}$ (49) (Fig. 2A panel 1). The technique was validated in two ways, both by immunofluorescence microscopy and by western blot (Fig. 2A panel 2). Confocal microscopy analysis detected streptavidin-546 punctate labelling in HeLa APEX2GFP-LC3B cells treated with biotin phenol but only in the presence of $\mathrm{H}_{2} \mathrm{O}_{2}$. Importantly, strepatvidin-546 puncta colocalised with or were in proximity to LC3B puncta (Fig. 2B). Biotinylated proteins were pulled-down with neutravidin and detected by western blot using streptavidin-HRP. Treatment with $\mathrm{H}_{2} \mathrm{O}_{2}$ increased the amount of biotinylated protein in HeLa APEX2-GFP-LC3B cells but not in HeLa GFP-LC3B cells (Fig. 2C). Panels 3 and 4 in Fig. 2A summarise the approach for the larger scale proximity proteomics experiment and hit validation, described in the next figures.

\section{APEX2 experiment: 407 positive hits were detected in proximity to LC3B}

We scaled up the experiment in order to detect the proteins proximal to LC3B. Both cell lines were incubated with biotin phenol for 30 minutes and $\mathrm{H}_{2} \mathrm{O}_{2}$ for 1 minute. HeLa GFPLC3B cells were used as a negative control for the APEX2 reaction. Following the reaction, cells were lysed and biotinylated proteins were isolated with a neutravidin pull-down. Before mass spectrometry analysis, the enrichment of biotinylated proteins in the lysates subjected to the neutravidin pull-down were verified by western blot using the streptavidin-HRP and an LC3B antibody. A much more intense smear of biotinylated proteins (Fig. 3A) and LC3B (Fig. 3B) was detected in HeLa APEX2-GFP-LC3B compared to HeLa GFP-LC3B.

The samples were processed for an on-bead digest and subsequently analysed by liquid chromatography tandem mass spectrometry (LC-MS/MS). Four experiments were performed (see details in Methods). Proteins were considered to be hits when they met the 
following criteria in at least two independent experiments, comparing results obtained with HeLa APEX2-GFP-LC3B versus HeLa GFP-LC3B: log2fold $>1$, MASCOT score $>50,>2$ peptides identified and unadjusted p-Value $<0.05$ (see Methods). Such analyses detected 407 proteins in proximity (distance less than $20 \mathrm{~nm}$ ) to APEX2-GFP-LC3B, with 237 of these having an FDR $<0.25$. Eighty-four percent of the hits were found in the four experiments and $97 \%$ were found in at least three experiments, demonstrating the reproducibility and robustness of our results (Fig. 3 CD).

\section{The 407 LC3B proximity proteins cover a broad range of pathways and cell localisations}

We ran a gene ontology (GO) analysis to identify the subcellular localisation of the 407 hits. We selected the GO terms with a $p$-Value $<0.05$ and grouped the hits into seven categories: cytoplasm/cytosol, cytoskeleton/cell junction, centrosome/spindle, endosome/trafficking, nucleus, endoplasmic reticulum (ER)/Golgi apparatus and preautophagosome/autophagosome (Fig. 4A and Figs. S1 and S2).

Using DAVID Bioinformatics Resources 6.8 (https://david.ncifcrf.gov/ $(54,55)$ ) to interrogate the KEGG pathways, followed by manual annotations using PubMed (https://pubmed.ncbi.nlm.nih.gov/) and UniProt (https://www.uniprot.org/ (56)), we determined that the 407 identified proteins are involved in a broad range of cellular pathways. These include "Endocytosis, trafficking, ubiquitination or proteasome", "RNA related proteins", "Cell cycle, mitosis and cytokinesis" and "Autophagy" (Figs. 4B and S3).

To determine which pathways were significantly enriched, we ran a "biological processes" GO analysis of the 407 hits. A subset of 109 proteins were enriched in 18 "biological processes" GO terms, with an FDR $<0.1$ and a fold enrichment $>2.5$ (Figs. 4C and S4). We organized these $18 \mathrm{GOs}$ into the four following categories: membrane trafficking (36.8\% of the hits), actin cytoskeleton organization $(19.1 \%)$, antigen processing and presentation of exogenous peptide antigen via MHC class II (11.8\%) and cell cycle/mitosis (44.1\%) (Figs. 4D and S5). 
To identify high confidence proximal proteins, we presented all 407 hits in a volcano plot, showing statistical significance (FDR score or $p$-Value) versus magnitude of change (fold change) between proteins detected in proximity to APEX2-GFP-LC3B and GFP-LC3B. Proximity proteins represented in these plots have a Mascot score $>50,>2$ peptides and a FDR score $<0.05$ (Fig. 4E) or p-Value $<0.05$ (Fig. S6B). Eighty-eight proximity proteins were detected (Fig. S6A).

\section{Thirty-four percent of the LC3B proximity proteins are already related to autophagy}

To validate our approach, we investigated which of the 407 LC3B proximity proteins have already been reported to play a role in autophagy or to be associated to the autophagosome and/or LC3.

This was determined through bioinformatic DAVID Pathway and GO localisation analyses (14 hits, Figs. 5A in yellow circle and S8) and through literature research of the high confidence hits revealed by the volcano plot representation (35 hits, Figs. 5A in green circle and S8). Further hits were found to overlap with four previous proteomic studies aiming at detecting proteins present in autophagosomes (Dengjel J. et al. (57), Mancias J. D. et al. (58), Gao W. et al. (59), Le Guerroué F. et al. (60)). In these studies, cells were treated in various ways to induce accumulation of autophagosomes. Quantitative proteomics was performed from purified autophagosomes (fractionation or immuno-purification) (57-59) or following an APEX2-GFP-LC3B pull-down from inside autophagosomes only (60). One hundred forty-eight of our 407 hits overlapped with at least one of these proteomic studies, including 68 further reported to play a role in autophagy or in its regulation (Figs. $\mathbf{5}$ in grey circle, S7 and S8). A literature search determined 41 additional hits related to autophagy that were not found with the above-cited methods (Figs. 5A inside red circle and S8).

We further interrogated the iLIR database (https://ilir.warwick.ac.uk (18)), containing all the putative canonical LIR-containing proteins identified in silico in the proteomes of eight model organisms, using the iLIR server (http://repeat.biol.ucy.ac.cy/iLIR/ (61)). Sixty-nine of our 407 hits were found in the iLIR database. Twenty-eight of these have a reported role in 
autophagy (Figs. 5A inside blue circle, S8 and S9), including 13 hits shown to associate (through co-immunoprecipitation or GST pull-down experiments) or colocalise with LC3B: ATG16L1 (A16L1), BAG6, BIRC6, CLU, EGFR, FKBP8, FYCO1, LRBA, NCOR1, SQSTM, TAX1BP1 (TAXB1), TBC1D5 (TBCD5), UBR4 (Figs. 5A, inside blue circle in bold, S8 and S9).

Within our autophagy hits, several core autophagy proteins or well-established regulators such as ATG3 (62), ATG7 (63), ATG9A (8), ATG16L1 (A16L1) (16), CALCOCO2 (CACO2) (64), FYCO1 (21), MTOR (65) and SQSTM (66), as well as LC3B (MLP3B) (10), were found (Fig. 5A). SQSTM/p62, known as the major receptor for selective autophagy (67), interacts directly with LC3B through its LIR (21), even in basal cell conditions (68). The presence of SQSTM was detected in the neutravidin pull-down (shown in Fig. 3) from HeLa APEX2-GFP-LC3B cells but not HeLa GFP-LC3B cells (Fig. 5B). SQSTM colocalised with GFP-LC3B puncta (Fig. 5C) in HeLa GFP-LC3B cells, ruling out non-specific detection of SQSTM with the APEX2 tag. Furthermore, SQSTM co-immunoprecipitated with LC3B in HeLa cells (expressing endogenous LC3B), using two distinct antibodies previously validated to correctly detect autophagosomes (69, 70) (Fig. 5D).

One of our high confidence hits was EEA1 (Early Endosome Antigen 1). This was revealed by the volcano plot representation and also detected in one of the four proteomic studies (57) (Fig. 5A in intersection between green and grey circles, Fig. S8). It is recruited to endosomal membranes by binding the phospholipid phosphatidylinositol 3-phosphate $(\mathrm{PI}(3) \mathrm{P})$ through its C-terminal FYVE domain (71) where it binds to its effector Rab5 $(72,73)$. It promotes sorting from the early endosome and early-late endosome fusion (74). Autophagic vacuoles can also fuse with EEA1-positive early endosomes, generating amphisomes (75). Moreover EEA1-positive endosome and autophagic vacuole fusion have been shown to be required for autophagosome maturation and autophagy (76). We confirmed the presence of EEA1 in the neutravidin pull-down (shown in Fig. 3) from HeLa APEX2-GFP-LC3B cells and not HeLa GFP-LC3B cells (Fig. 5E). GFP-LC3B and EEA1 colocalisation was also detected (Fig. 5F). 
Therefore, at least 141 LC3B proximity proteins, $34.6 \%$ of our total 407 hits, were found to be related to autophagy (Fig. 5A and Figs. S8 and S10), including several proteins with putative LIR domains, validating our method. Interestingly 30 of these 141 autophagyrelated hits were shown to associate (through co-immunoprecipitation or GST-pull-down experiments) or colocalise with LC3B (Figs. 5A, in bold and S8). Our study also revealed that 111 of the 141 autophagy-related hits have no published interaction or colocalisation with LC3 to our knowledge (others than in the four proteomic studies) (Fig. S8).

The endosomal protein GAPD1 has a putative LIR domain and is in proximity to LC3B Among our 407 hits, 41 have not been reported to associate with LC3 or to be linked to autophagy, but have a putative LIR domain (Fig. 6A). Twenty-nine of these proteins were found to be part of "endocytosis or membrane trafficking" in the DAVID Pathway analysis or localised on endosomes in the GO localisation analyses (Figs. 6B and S11). "Endosomal trafficking" is one of the four pathways enriched in the GO "biological processes" analysis (Fig. 4D). One of these proteins is GAPD1 or GAPVD1 (GTPase-activating protein and VPS9 domain-containing protein 1), also called RAP6 (Rab5-activating protein 6) and GAPex5. As seen in the diagram Fig. 6C, obtained by the software STRING, GAPD1 is an endosomal protein that biologically interacts with a number of others such as Rab5A and B. It plays a role in constitutive and regulated endocytosis and is involved in the regulation of receptor-mediated endocytosis (77). GAPD1 was detected by western blot in the neutravidin pull-down from the HeLa APEX2-GFP-LC3B cells and not HeLa GFP-LC3B cells (Fig. 6D). We then confirmed GAPD1 colocalisation with GFP-LC3B puncta (Fig. 6E). Furthermore, GAPD1 coimmunoprecipitated with endogenous LC3B in HeLa cells (Fig. 6F).

The translation initiation factor elF2D has a putative LIR domain and is associated with LC3B

Five of the LC3B proximity proteins with putative LIR domains, which have not been previously reported to bind LC3, are part of the category "RNA related proteins" in the DAVID 
Pathway analysis. These are AASD1, CNO10, EIF2D, PRC2C and R3HD1 (Figs. 6AG, S3 and S11). EIF2D is an initiation factor of translation, part of the 435 preinitiation complex. Interestingly, the following hits were not found in the ILIR database, but are also initiation factors: elF3A, elF3B, elF3C, elF3G, elF3H, elF3I (78) (Fig. S11). EIF2D colocalised with GFP-LC3B puncta (Fig. 6H) and co-immunoprecipitated with endogenous LC3B (Fig. 6IJ), validating the APEX2 result. While eIF2A phosphorylation has previously been shown to be necessary for the stimulation of autophagy, it is through transcription of several autophagy genes (SQSTM, LC3B, BECN1, ATG3, ATG7) $(79,80)$. Therefore, our results, revealing a novel LC3B-elF2D interaction, suggest a novel relationship between the initiation of translation and LC3B. 


\section{Discussion}

In this study, we used peroxidase-mediated proteomic mapping in live cells combined with unlabelled mass spectrometry to determine the proteins in proximity $(\leq 20 \mathrm{~nm})$ to LC3B in HeLa cells cultured in full medium. Four hundred and seven LC3B proximity proteins were detected. One hundred forty one of these proteins, or $34.6 \%$ of the total hits, have been previously reported as related to the autophagy process or its regulation (Fig. 5A), validating our approach. These include several core autophagy drivers or regulators. Sixty-eight of these 141 autophagy-related proteins have been determined to be present in the autophagosome or associated with LC3 in at least one of four previous proteomic studies (57-60). Moreover, 30 of the 141 proteins have already been shown to associate with LC3 through coimmunoprecipitation, GST pull-down or colocalisation studies (Fig. 5A). Using coimmunofluorescence analysed by confocal microscopy and co-immunoprecipitation experiments, we validated two of these proteins in our culture conditions: the autophagy receptor SQSTM (or p62) (67) (Fig. 5BCD), containing an $\operatorname{LIR}(18,21)$, and the sorting endosome marker EEA1 (72, 75) (Fig. 5EF). Twenty-eight of the 141 autophagy-related hits were found in the iLIR database, including 13 with demonstrated LC3B association (Fig. 5A in bold in the blue circle), further validating our approach.

Interestingly, for 53 hits found in at least one of the four proteomic studies and shown to play a role in autophagy, there is no association with LC3 further reported in the literature (through co-immunoprecipitation or GST pull-down or colocalisation) (Fig. S7). It is, for example, the case for ROCK1 and ROCK2, two key protein kinase regulators of the actin cytoskeleton and cell polarity (81). Furthermore, 69 of the total 407 hits were found in the iLIR database, including 56 proteins that have not, to our knowledge, been shown to be associated with LC3 (through co-immunoprecipitation or GST-pull-down or colocalisation) (Figs. 6A and S9). On the other hand, 41 proteins with a putative LIR domain have not been reported to be related to autophagy (Fig. 6A). These findings suggest that a direct interaction between some of these proteins and LC3B may regulate an unknown function. 
Previous proteomic studies aimed to determine proteins present in the autophagosome and thus were performed from enriched autophagosome from cells that were serum or amino acid-deprived or treated with inhibitors to trigger stress-induced autophagy or inhibit autophagic flux $(60,82)$. Interestingly, a LC3-APEX2 screen (60) has recently been published, and 54 of our hits are common with this study (Fig. S7). This study determined LC3B proximity proteins inside the autophagosome. Our study has a distinct focus from these previous studies, which is to uncover the LC3B global interactome, irrespective of LC3B localisation (inner or outer membrane of the autophagosome or other cell location). We have determined the LC3B interactors from whole cell lysates and in basal cell condition (using cells maintained in full medium and without inhibitors). Thus our experimental conditions allow the detection of proteins in proximity to LC3B potentially involved in the three primary types of autophagy: microautophagy (83), macroautophagy (6), and CMA (84), but also in noncanonical autophagy or even functions unrelated to autophagy $(26,35,38,85,86)$. Additional LC3-ylated proteins (that we recently described (47)) may also have been uncovered.

Accordingly, we found some novel LC3B interactors, involved in a broad range of pathways and cell localisation (Fig. 4). The pathway functions include membrane trafficking, cytoskeleton rearrangement, antigen processing and presentation of exogenous peptide antigens, RNA-related proteins, cell cycle/mitosis/cytokinesis and nuclear proteins. Interestingly, a pool of LC3 has recently been shown to localise to the nucleus and initiate autophagy upon redistribution to the cytoplasm $(63,87-89)$. Over $16 \%$ of the 407 proteins detected in proximity to LC3B have a reported nuclear localisation (Fig. 4A), and some could be novel partners or regulators of LC3B nucleo-cytoplasm shuttling. Moreover, LC3 has been reported to associate with nuclear complexes $(45,90)$.

A substantial proportion of our hits were part of pathways we regrouped under "actin cytoskeleton organisation/focal adhesion/ECM/cell junction" (Fig. 4). Among these, two proteins of the alpha-actinin family (ACTN1 and ACTN4) are cytoskeletal actin-binding proteins with an important role in the structure and regulation of cytoskeleton organization and 
muscle contraction (91). These two proteins were not found in the iLIR database. ACTN4, but not ACTN1, has been described in autophagy (92) although with no interaction with LC3B.

Another group of our identified LC3B interactors were previously reported to be part of the membrane trafficking localisation (24.7\%) or pathway (25.9\% of the hits) (Fig. 4AB). It is one of the four enriched pathway categories in the GO "biological functions" analysis and accounts for $36.8 \%$ of the hits enriched (Fig. 4D). This could reflect the highly dynamic membrane trafficking which depends on various endomembranes connecting with each other, through maturation or fusion. Nevertheless, 53 of these proteins have a putative LIR domain, including 11 reported associated with LC3, reinforcing the possibility that they are true LC3B interactors (Fig. S12). Moreover, 29 proteins with a putative LIR domain and not previously reported to be related to autophagy are membrane trafficking proteins or have been found localised to endomembranes (Fig. 6B). We have validated one of these proteins, GAPD1, a regulator of endocytosis that exhibits GEF activity specific for RAB5 and GAP activity specific for RAS (77) (Fig. 6DEF).

Eleven-point nine percent of the 407 hits are part of "RNA binding proteins" as detected by the DAVID database (Fig. 4B). Several of these proteins have not been reported to be related to autophagy and have a putative LIR domain (Fig. 6G). Of these, we have validated the translation factor EIF2D (Fig. 6HI). The fact that several other initiation factors of translation, all part of the $43 S$ preinitiation complex (78), were also detected in proximity to LC3B strongly suggest a previously unrecognised relationship between LC3B and this complex.

This study has uncovered the LC3B proximity proteome in cells in normal growth culture. We have discovered a number of novel LC3B proximity proteins, opening the way to a better understanding of LC3B's role in autophagy and possibly functions not related to autophagy. 


\section{Authors contribution}

MN designed, performed and analyzed most of the experiments and wrote the manuscript. AA generated and characterized the constructs and cell lines. FM performed some of the experiments and provided comments on the manuscript. ADD performed the bioinformatic analyses. VR performed and analyzed the proteomics. JB performed and analyzed the electronic microscopy. TN and RK contributed to the experimental design, to the interpretation of the results and provided comments on the manuscript. PC directed the proteomic experiments, performed the related bioinformatic analyses and provided comments on the manuscript. SK conceived and directed the project, designed the experiments and wrote the manuscript.

\section{Acknowledgements}

We thank Sara Farrah Heuss for her assistance in the preliminary APEX2 experiments and Brynna Hoggard for the critical reading of the manuscript.

This work was supported by the Medical Research Council (MR/R009732/1), Rosetrees Trust (M314) and Barts and The London Charity (MGU0511).

\section{Declaration of interests}

The authors declare no competing interest. 


\section{Titles and legends}

\section{Fig. 1: APEX2-GFP-LC3B labels autophagosomes}

(A) Schematic of APEX2-GFP-LC3B and GFP-LC3B constructs.

(B) Expression of LC3B and the loading control HSC70 was evaluated in HeLa GFP-LC3B and HeLa APEX2-GFP-LC3B lysates by western blot.

(C) Confocal sections of HeLa APEX2-GFP-LC3B cells treated with bafilomycin A1 (baf A1; $10 \mathrm{nM})$, torin $1(250 \mathrm{nM})$ or a combination of both for 3 hours. GFP-LC3B (green), LAMP1 (red) and Hoechst (blue). Colocalisation appears as yellow puncta. Scale bar, $10 \mu \mathrm{m}$.

(D) HeLa APEX2-GFP-LC3B cells were treated for 3 hours with $10 \mathrm{nM}$ bafilomycin A1 and $250 \mathrm{nM}$ torin 1 prior to sample processing for electron microscopy with or without DAB substrate. Scale bar, $2 \mu \mathrm{m}$. Higher magnifications of boxes 1, 2 and 3 are shown below. Scale bar, $0.5 \mu \mathrm{m}$.

\section{Fig. 2: APEX2 workflow and validation of the method}

(A) Diagram showing the experimental workflow. 1) HeLa cells expressing APEX2-GFP-LC3B or GFP-LC3B were incubated with biotin phenol for 30 minutes and with $\mathrm{H}_{2} \mathrm{O}_{2}$ for 1 minute, allowing biotinylation of proteins in proximity to APEX2-GFP-LC3B. 2) The technique was validated by immunofluorescence and western blot with streptavidin isolation. 3) The experiment was scaled up for proteomic analysis. Here biotinylated proteins were pulled-down with neutravidin and analysed by mass spectrometry followed by a range of bioinformatic and statistical analyses. 4) Hits were validated based on literature research, interrogation of the LIR database and further technical validation for selected proteins.

(B) Confocal sections of HeLa APEX2-GFP-LC3B cells incubated with biotin phenol for 30 minutes with or without a subsequent 1 minute incubation with $\mathrm{H}_{2} \mathrm{O}_{2}$. GFP-LC3B (green), Streptavidin-546 (red) and DAPI (blue). Colocalisation appears as yellow puncta. Scale bar, $10 \mu \mathrm{m}$.

(C) Levels of streptavidin-HRP evaluated by western blot in HeLa GFP-LC3B and HeLa APEX2-GFP-LC3B neutravidin pull-down eluates following the APEX2 assay (left) or 
corresponding lysates (right). Cells were incubated with biotin phenol for 30 minutes and with or without $\mathrm{H}_{2} \mathrm{O}_{2}$ for 1 minute.

Fig. 3: 407 hits in proximity to APEX2-GFP-LC3B were detected by proteomics

(A, B) Before the mass spectrometry analyses, levels of biotinylated proteins detected by streptavidin-HRP (A) and LC3B (B) were evaluated by western blot in HeLa GFP-LC3B and HeLa APEX2-GFP-LC3B neutravidin pull-down eluates following the APEX2 assay. Cells were incubated with biotin phenol 30 minutes and $\mathrm{H}_{2} \mathrm{O}_{2}$ for 1 minute. The experiment was performed in duplicates.

(C) Pie graph showing the percentage of the 407 proteomic hits overlapping between 2, 3 or 4 experiments.

(D) Heat map representation of the 407 hits in HeLa APEX2-GFP-LC3B cells and HeLa GFPLC3B cells in each of the four experiments. Proteins were selected if log2 HeLa APEX2-GFPLC3B compared to HeLa GFP-LC3B >1, MASCOT score $>50,>2$ peptides identified and pValue $<0.05$

Fig. 4: The 407 hits grouped in localisations, pathways, gene ontology enrichment and high confidence hits

(A) Pie graph representation of different localisations found by gene ontology (GO) analysis. The percentage of proteins in each localisation is indicated.

(B) Bar graph showing the different pathways found by an analysis with DAVID software (https://david.ncifcrf.gov/) completed with a search by PubMed and UniProt. The percentage of proteins in each pathway is indicated.

(C, D) Enrichment analysis of "biological processes" GO analysis.

(C) Volcano Plot analysis of "biological processes" GO analysis. Proteins were selected following $p$-Value $<0.05$ and MASCOT score $>50$. The names and the red points are for gene ontologies with FDR $<0.1$ and fold enrichment $>2.5$. 
(D) Pie graph representation of the different "biological processes" genes found by GO and grouped into four categories. The percentage of proteins in each group is indicated.

(E) Volcano plot revealing the "high-confidence hits" representing the FDR in function of the Log2fold of the hits obtained in HeLa APEX2-GFP-LC3B cells compared to HeLa GFP-LC3B cells. Hits with a Mascot score $>50,>2$ peptides and a FDR score $<0.05$ were selected.

\section{Fig. 5: Autophagy-related hits}

(A) List of the 141 autophagy-related hits. Proteins in bold have been shown associated with LC3 (through co-immunoprecipitation, pull-down or co-immunofluorescence). Green circle: high confidence hits revealed by the volcano plot representation in Fig. 4E. Blue circle: containing a putative LIR. Yellow circle: present in pathways of autophagy (DAVID search) or in the GO localisation in autophagosome. Grey circle: present in at least one of the four proteomics studies analysed (57-60). Red circle: present in literature only.

(B, C, D) Validation of LC3B-SQSTM proximity.

(B) Western blot for SQSTM from neutravidin pull-down eluates following the APEX2 assay in HeLa APEX2-GFP-LC3B and HeLa GFP-LC3B cells.

(C) Confocal sections of HeLa GFP-LC3B cells. GFP-LC3B (green), SQSTM (red) and DAPI (blue). Colocalisation appears in yellow. Scale bar, $10 \mu \mathrm{m}$.

(D) Western blots for LC3B and SQSTM following immunoprecipitation of endogenous LC3B in HeLa cells performed in duplicate with an Abcam antibody (Ab1, left side of the blot) and a Cell Signalling Technology antibody, clone D11 (Ab2, right side of the blot) or corresponding IgG control. Total levels of LC3B-I and II and SQSTM in the cell lysates are also shown.

(E, F) Validation of LC3B-EEA1 proximity.

(E) Western blot for EEA1 from neutravidin pull-down eluates following the APEX2 assay in HeLa APEX2-GFP-LC3B and HeLa GFP-LC3B cells.

(F) Confocal sections of HeLa GFP-LC3B cells. GFP-LC3B (green), EEA1 (red) and DAPI (blue). Colocalisation appears in yellow. Scale bar, $10 \mu \mathrm{m}$. 
Fig. 6: Hits not reported related to autophagy but with putative LIR domains and validation: trafficking pathway and RNA binding/Initiation of translation factors

(A) Heat map of the 41 hits not previously shown to be related to autophagy and found in the iLIR database. For each protein, the log2fold HeLa APEX2-GFP-LC3B compared to HeLa GFP-LC3B is indicated with a matching colour gradient.

(B) List of the hits from (A) reported to be involved in endocytosis/membrane trafficking pathways in the DAVID pathway database (green circle), in the "biological processes" GO analysis (orange circle) or localised to endosomes as determined by the GO localisation analysis (blue circle).

(C) GAPD1 network obtained by STRING software.

(D, E, F) Validation of LC3B-GAPD1 proximity

(D) Western blot for GAPD1 from neutravidin pull-down eluates following the APEX2 assay in HeLa APEX2-GFP-LC3B and HeLa GFP-LC3B cells.

(E) Confocal sections of HeLa GFP-LC3B cells. GFP-LC3B (green), GAPD1 (red) and DAPI (blue). Colocalisation appears in yellow. Scale bar, $10 \mu \mathrm{m}$.

(F) Western blots for LC3B and GAPD1 following immunoprecipitation of endogenous LC3B in HeLa cells performed in duplicate with an Abcam antibody (Ab1, left side of the blot) and a Cell Signalling Technology antibody, clone D11 (Ab2, right side of the blot) or corresponding IgG control. Total levels of LC3B-I and II and GAPD1 in the cell lysates are also shown.

(G) List of the proteins not previously shown to be related to autophagy and found in the iLIR database (from Fig. 6A) and reported to be involved in RNA binding, degradation or translation in the DAVID pathway database.

(H) Confocal sections of HeLa GFP-LC3B cells. GFP-LC3B (green), elF2D (red) and DAPI (blue). Colocalisation appears in yellow. Scale bar, $10 \mu \mathrm{m}$.

(I) Western blots for LC3B and elF2D following immunoprecipitation of endogenous LC3B in HeLa cells performed in duplicate with an Abcam antibody (Ab1, left side of the blot) and a Cell Signalling Technology antibody, clone D11 (Ab2, right side of the blot) or corresponding IgG control. Total levels of LC3B-I and II and elF2D in the cell lysates are also shown. 
bioRxiv preprint doi: https://doi.org/10.1101/2021.10.08.463639; this version posted October 9, 2021. The copyright holder for this preprint

(which was not certified by peer review) is the author/funder. All rights reserved. No reuse allowed without permission.

Nollet et al, 21

(J) Western blots for elF2D and HSC70 from a large amount of protein lysate. 


\section{Methods}

\section{Generation of HeLa cells stably expressing APEX2-GFP-LC3B}

The APEX2 coding and linker peptide sequence was PCR-amplified from pcDNA3 Connexin43-GFP-APEX2, a gift from Alice Ting (Addgene plasmid \# 49385; http://n2t.net/addgene:49385; RRID:Addgene_49385) (93), using the primers 5'-Pacl-APEX2 (5'-GACTTAATTAAGCCACCATGGGAAAGTCTTACCCAACTGTG-3') and 3'-Xbal-linkerAPEX2 (5'-GACTCTAGATCCGGAGCCCGAGCCCGAGGTCGAGCCCGAGCCCTTGGCAT CAGCAAACCCAAGC-3'). The resulting PCR product was inserted into pLVpuro-CMV-NEGFP (Addgene plasmid \# 122848 ; http://n2t.net/addgene:122848; RRID:Addgene_122848) (94) by restriction-based cloning, between the Pacl and Xbal sites, to generate pLVpuro-CMVN-APEX2-EGFP. Gateway recombination of the destination vector pLVpuro-CMV-N-APEX2EGFP with the entry clone pDONR223 LC3B WT (Addgene plasmid \# 123072; http://n2t.net/addgene:123072; RRID:Addgene_123072) (94) was performed using LR clonase II enzyme mix (ThermoFisher Scientific, 11791020), to generate pLVpuro-CMVAPEX2-EGFP-LC3B. Lentiviral packaging and stable cell line generation was then performed as previously described (94) using pLVpuro-CMV-APEX2-EGFP-LC3B as the transfer plasmid and infecting wild type HeLa cells with the resulting lentiviral particles. A clonal HeLa cell line expressing moderate levels of APEX2-EGFP-LC3B was isolated by limit dilution prior to expansion and use in all experiments described. As a control, wild type HeLa cells were infected in parallel with lentivirus encoding CMV-driven EGFP-LC3B generated previously (94) and expanded as a pool.

\section{Cell culture}

HeLa cells were cultured in Dulbecco's Modified Eagle's Medium (DMEM, Gibco) supplemented with $10 \%$ fetal bovine serum (FBS, Gibco). For the HeLa cells infected with GFP-LC3B or APEX2-GFP-LC3B lentivirus, puromycin $(2 \mu \mathrm{g} / \mathrm{ml}$, Sigma Aldrich) was added in the medium. The cells were grown in a humidified atmosphere with $5 \% \mathrm{CO}_{2}$ at $37^{\circ} \mathrm{C}$. 


\section{Western blot}

Cells were lysed in sample buffer (Thermofisher) supplemented with dithiothreietal (DTT, Fisher), sonicated and boiled at $99^{\circ} \mathrm{C}$ for $5 \mathrm{~min}$. Samples were loaded on 4-12\% Novex BisTris gels (Invitrogen). Separated proteins were transferred onto $0.45 \mu \mathrm{m}$ nitrocellulose transfer membranes (VWR International). Protein loading and transfer quality were checked by staining with Ponceau S. Membranes were blocked in TBS with 4\% BSA, then probed with the following primary antibodies: EEA1 (Santa cruz), elF2D (invitrogen), GAPD1 (Novusbio), HSC70 (Santa Cruz), LC3B (Clone D11, Cell Signaling Technology), and SQSTM (Cell Signaling Technology) at a 1:1000 dilution and Streptavidin HRP (Millipore) at a 1:10,000 dilution. Membranes were incubated overnight at $4^{\circ} \mathrm{C}$, followed by a one hour incubation in the appropriate secondary antibodies coupled to peroxidase (1:1000, Biorad). Proteins were detected by enhanced chemiluminescence detection (ECL, GE Healthcare). Densitometric analyses of immunoblots were performed using ImageJ 1.50i (National Institute of Health).

\section{Immunofluorescence and confocal microscopy}

Cells were plated onto coverslips in 24-well plates (50000 cells per well). For most experiments, cells were fixed in $2 \%$ paraformaldehyde for 15 minutes. Free aldehydes were quenched with $50 \mathrm{mM} \mathrm{NH}_{4} \mathrm{Cl}$ in PBS for 10 min. Fixed cells were blocked and permeabilized in PBS with $3 \%$ BSA and $0.1 \%$ Triton $\mathrm{X}-100$ for 15 minutes. Cells were incubated with the following primary antibodies: elF2D (invitrogen), GAPD1 (RAP6 Novusbio), LC3B (Catalogue number 2775S, Cell Signaling Technology), and SQSTM (Cell Signaling Technology) at a 1:100 dilution 1:100 and streptavidin-546 (Life technologies) at a 1:500 dilution for 30 minutes. Cells were washed three times in PBS, followed by a 20 minutes incubation with the appropriate secondary antibodies coupled to a fluorochrome Alexa 555 (1:500, Life technologies). Cells were washed three times in PBS and once in water and mounted onto slides with Prolong ${ }^{\text {TM }}$ Gold Antifade Mountant with DAPI (Molecular probes, Life technologies). Images were acquired using a confocal laser scanning microscope (LSM710; Carl Zeiss, Inc.) 
equipped with a 63x1,4 NA Plan-Apochromat oil immersion objective. Alexa 488 was excited with the $488 \mathrm{~nm}$ line of an Argon laser, Alexa 555 was excited with a $543 \mathrm{~nm}$ HeNe laser.

For immunofluorescence using the anti-LAMP1 antibody (1:1,000, BD Biosciences, Catalogue number 555798) we used a protocol previously described (94) and imaged with a Leica SPE confocal microscope using a 1.3 NA 63x oil immersion objective.

All image fields were chosen arbitrarily based on DAPI (4,6-diamidino-2-phenylindole) staining and images were taken in unsaturated conditions.

\section{Electron microscopy}

Transmission electron microscopy sample preparation and imaging was performed as previously described (94) with some modifications. The DAB substrate mix was freshly prepared using 3.5 mM DAB (3,3'-Diaminobenzidine tetra-HCl, obtained from TAAB UK) and $0.02 \% \mathrm{H}_{2} \mathrm{O}_{2}$ (Sigma) in $50 \mathrm{mM}$ Tris- $\mathrm{HCl}$ at a $\mathrm{pH}$ of 7.6 . Immediately following glutaraldehyde fixation and rinsing in $0.1 \mathrm{M}$ sodium cacodylate, samples were incubated with or without the DAB substrate mix for 3 minutes at room temperature. To stop the reaction, samples were rinsed in $50 \mathrm{mM}$ Tris- $\mathrm{HCl}$ buffer followed by $0.1 \mathrm{M}$ sodium cacodylate. Samples were then fixed with osmium and processed as previously described (94), but with the omission of tannic acid and sodium sulphate incubations.

\section{Co-Immunoprecipitation}

Cells were lysed in RIPA buffer (Merck) supplemented with phosphatase and protease inhibitors. Lysates were centrifuged at $10000 \mathrm{rpm}$ for 10 minutes at $4^{\circ} \mathrm{C}$. The supernatant was precleared with beads (Protein G sepharose ${ }^{\mathrm{TM}} 4$ flast flow, GE Healthcare) for 1 hour at $4^{\circ} \mathrm{C}$ on a rotating wheel. Beads were removed and $4 \mu \mathrm{g}$ LC3B antibody (Abcam or clone D11 Cell Signalling Technology) or IgG was added and rotated overnight at $4^{\circ} \mathrm{C}$. The following day, beads were added and rotated for 1 hour at $4^{\circ} \mathrm{C}$. Beads were washed 3 times with RIPA and sample buffer supplemented with DTT was added. 


\section{APEX2 experiment}

Five million cells were plated in $100 \times 17 \mathrm{~mm}$ dishes in full media containing $7 \mu \mathrm{M}$ Heme (Sigma). After 24 hours, the cells were treated with $500 \mu \mathrm{M}$ biotin tyramide (biotin phenol; Iris Biotech) for 30 minutes at $37^{\circ} \mathrm{C}$ and exposed to $1 \mathrm{mM}$ freshly prepared hydrogen peroxide for 1 minute. The biotinylation reaction was quenched by 3 washes of a stop solution prepared with $10 \mathrm{mM}$ 6-Hydroxy-2,5,7,8-tetramethylchroman-2-carboxylic acid (Trolox; Sigma), $20 \mathrm{mM}$ sodium Ascorbate and $10 \mu \mathrm{M}$ sodium azide in PBS. Cells were lysed at $4^{\circ} \mathrm{C}$ in RIPA buffer supplemented with $10 \mathrm{mM}$ sodium azide and protease inhibitors. Lysates were centrifuged at $14000 \mathrm{rpm}$ for 15 minutes at $4^{\circ} \mathrm{C}$ and protein concentration determined with a Pierce ${ }^{\mathrm{TM}} \mathrm{BCA}$ Protein Assay Kit (Life Technologies). High capacity neutravidin beads (Life Technologies) were added to the lysates and rotated overnight at $4^{\circ} \mathrm{C}$. Beads were washed 3 times with RIPA. For western blot, sample buffer supplemented with DTT was added. For mass spectrometry, beads were washed with $25 \mathrm{mM}$ ammonium bicarbonate buffer 3 times, centrifuged at $14000 \mathrm{rpm}$ and frozen at $-80^{\circ} \mathrm{C}$. Three independent experiments were performed, including one experiment in duplicate (called experiment 3 and 4 ), thus four replicates were produced.

\section{Mass spectrometry}

Proteomics experiments were performed using mass spectrometry as previously described $(95,96)$. Immunoprecipitated (IP) protein complex beads were digested into peptides using trypsin and peptides were desalted using $\mathrm{C} 18+$ carbon top tips (Glygen corparation, TT2MC18.96) and eluted using $70 \%$ acetonitrile (ACN) with $0.1 \%$ formic acid. Dried peptides were dissolved in $0.1 \%$ TFA and analysed by ultimate 3000 RSL nanoflow liquid chromatograph coupled on-line to a $Q$ Exactive plus mass spectrometer (Thermo Fisher Scientific). Gradient elution was from $3 \%$ to $35 \%$ buffer B over 120 minutes at a flow rate of $250 \mathrm{~nL} / \mathrm{min}$ with buffer $\mathrm{A}$ being used to balance the mobile phase. Buffer $A$ was $0.1 \%$ formic acid in water and $\mathrm{B}$ was $0.1 \%$ formic acid in $\mathrm{ACN}$. The mass spectrometer was controlled by Xcalibur software (version 4.0) and operated in the positive ionisation mode. The spray voltage 
was $1.95 \mathrm{kV}$ and the capillary temperature was set to $255^{\circ} \mathrm{C}$. The Q-Exactive plus was operated in a data-dependent mode with one survey MS scan followed by 15 MS/MS scans. The full scans were acquired in the mass analyser at $375-1500 \mathrm{~m} / \mathrm{z}$ with a resolution of 70000 , and the MS/MS scans were obtained with a resolution of 17500.

MS raw files were converted into Mascot Generic Format using Mascot Distiller (version 2.7.1) and compared to the SwissProt database using the Mascot search daemon (version 2.6.0) with FDR of $\sim 1 \%$ and restricted to human entries. Allowed mass windows were $10 \mathrm{ppm}$ and $25 \mathrm{mmu}$ for parent and fragment mass to charge values, respectively. Variable modifications included in searches were oxidation of methionine, pyro-glu (N-term) and phosphorylation of serine, threonine and tyrosine. The mascot result (DAT) files were extracted into Excel files for further normalisation, quantitative label-free analysis and statistical analysis as described previously $(49,96)$.

\section{Bioinformatic analyses}

\section{Statistical analyses}

Statistical analyses were performed within the R (v3.6.1) statistical computing environment (98). Following sum scaling to normalise the protein quantification data, missing values were replaced by the minimum observed value within each sample. For differential analysis, proteins were first filtered on identification confidence criteria (Mascot score $>50,>2$ detected peptides per protein; in APEX2 only comparisons, any proteins detected in GFP controls were removed) and then the log 2 transformed average fold change was calculated between the conditions. Unpaired, two-tailed t tests were used to assess significance in the proteomics data. Where applicable, $p$-Values were adjusted for multiple testing using the BenjaminiHochberg method (99). The analyses were visualized using a combination of individual $R$ packages, namely: ggplot2, gplots, reshape2, Hmisc, readXL and ggrepel (100-105). 
For gene ontology analysis, proteins differentially expressed between conditions (at $p$-Value $<0.05)$ were grouped into gene ontologies as annotated in UniProt and DAVID informatics curated databases (Biological processes/cellular compartment/molecular function) (54-56). To infer ontology enrichment across sets of samples, a hypergeometric test was used followed by Benjamini-Hochberg multiple testing correction.

For literature validation analysis, supplementary data from four studies (57-60) that had previously performed autophagy-associated proteomics studies were extracted. These datasets were merged and matched against the APEX2-enriched proteins identified in this study that met the following criteria: $\geq 2$ Log2 fold change in abundance compared to GFP controls, with a Mascot score $>50$.

STRING protein-protein interaction (PPI) analyses

STRING (Search Tool for the Retrieval of INteracting Genes) V11 (https://string-db.org/) was used to study the PPI network. The settings "multiple proteins" and organism "Homo sapiens" were selected (106).

DAVID analyses

DAVID Bioinformatics Resources 6.8, NIAID/NIH (https://david.ncifcrf.gov/) was used to identify the KEGG (Kyoto Encyclopedia of Genes and Genomes) pathways. KEGG is a collection of databases. The settings "UniProt ID" and "gene list" were selected (55). 


\section{References}

1. Kirisako, T., Baba, M., Ishihara, N., Miyazawa, K., Ohsumi, M., Yoshimori, T., Noda, T., and Ohsumi, Y. (1999) Formation Process of Autophagosome Is Traced with Apg8/Aut7p in Yeast. J Cell Biol. 147, 435-446

2. Parzych, K. R., and Klionsky, D. J. (2014) An Overview of Autophagy: Morphology, Mechanism, and Regulation. Antioxid Redox Signal. 20, 460-473

3. Mizushima, N. (2007) Autophagy: process and function. Genes Dev. 21, 2861-2873

4. Dunn, W. A. (1990) Studies on the mechanisms of autophagy: formation of the autophagic vacuole. The Journal of Cell Biology. 110, 1923-1933

5. Lee, Y.-K., and Lee, J.-A. (2016) Role of the mammalian ATG8/LC3 family in autophagy: differential and compensatory roles in the spatiotemporal regulation of autophagy. $B M B$ Rep. 49, 424-430

6. Mizushima, N., Levine, B., Cuervo, A. M., and Klionsky, D. J. (2008) Autophagy fights disease through cellular self-digestion. Nature. 451, 1069-1075

7. White, E. (2015) The role for autophagy in cancer. J Clin Invest. 125, 42-46

8. Li, X., He, S., and Ma, B. (2020) Autophagy and autophagy-related proteins in cancer. Mol Cancer. 10.1186/s12943-020-1138-4

9. White, E. J., Martin, V., Liu, J.-L., Klein, S. R., Piya, S., Gomez-Manzano, C., Fueyo, J., and Jiang, H. (2011) Autophagy regulation in cancer development and therapy. Am J Cancer Res. 1, 362372

10. Kabeya, Y., Mizushima, N., Ueno, T., Yamamoto, A., Kirisako, T., Noda, T., Kominami, E., Ohsumi, Y., and Yoshimori, T. (2000) LC3, a mammalian homologue of yeast Apg8p, is localized in autophagosome membranes after processing. EMBO J. 19, 5720-5728

11. Weidberg, H., Shpilka, T., Shvets, E., Abada, A., Shimron, F., and Elazar, Z. (2011) LC3 and GATE-16 $\mathrm{N}$ termini mediate membrane fusion processes required for autophagosome biogenesis. Dev Cell. 20, 444-454

12. Takeshige, K., Baba, M., Tsuboi, S., Noda, T., and Ohsumi, Y. (1992) Autophagy in yeast demonstrated with proteinase-deficient mutants and conditions for its induction. J. Cell Biol. 119, 301-311

13. Dooley, H. C., Razi, M., Polson, H. E. J., Girardin, S. E., Wilson, M. I., and Tooze, S. A. (2014) WIPI2 links LC3 conjugation with PI3P, autophagosome formation, and pathogen clearance by recruiting Atg12-5-16L1. Mol. Cell. 55, 238-252

14. Tanida, I., Ueno, T., and Kominami, E. (2004) LC3 conjugation system in mammalian autophagy. Int. J. Biochem. Cell Biol. 36, 2503-2518

15. Ichimura, Y., Kirisako, T., Takao, T., Satomi, Y., Shimonishi, Y., Ishihara, N., Mizushima, N., Tanida, I., Kominami, E., Ohsumi, M., Noda, T., and Ohsumi, Y. (2000) A ubiquitin-like system mediates protein lipidation. Nature. 408, 488-492

16. Fujita, N., Itoh, T., Omori, H., Fukuda, M., Noda, T., and Yoshimori, T. (2008) The Atg16L complex specifies the site of LC3 lipidation for membrane biogenesis in autophagy. Mol. Biol. Cell. 19, 2092-2100

17. Sou, Y., Waguri, S., Iwata, J., Ueno, T., Fujimura, T., Hara, T., Sawada, N., Yamada, A., Mizushima, N., Uchiyama, Y., Kominami, E., Tanaka, K., and Komatsu, M. (2008) The Atg8 conjugation system is indispensable for proper development of autophagic isolation membranes in mice. Mol Biol Cell. 19, 4762-4775

18. Jacomin, A.-C., Samavedam, S., Promponas, V., and Nezis, I. P. (2016) iLIR database: A web resource for LIR motif-containing proteins in eukaryotes. Autophagy. 12, 1945-1953

19. Birgisdottir, Å. B., Lamark, T., and Johansen, T. (2013) The LIR motif - crucial for selective autophagy. J Cell Sci. 126, 3237-3247

20. Wild, P., McEwan, D. G., and Dikic, I. (2014) The LC3 interactome at a glance. J. Cell. Sci. 127, 3-9 
21. Pankiv, S., Clausen, T. H., Lamark, T., Brech, A., Bruun, J.-A., Outzen, H., Øvervatn, A., Bjørkøy, G., and Johansen, T. (2007) p62/SQSTM1 Binds Directly to Atg8/LC3 to Facilitate Degradation of Ubiquitinated Protein Aggregates by Autophagy. J. Biol. Chem. 282, 24131-24145

22. Ichimura, Y., Kumanomidou, T., Sou, Y., Mizushima, T., Ezaki, J., Ueno, T., Kominami, E., Yamane, T., Tanaka, K., and Komatsu, M. (2008) Structural basis for sorting mechanism of p62 in selective autophagy. J. Biol. Chem. 283, 22847-22857

23. Kraft, C., Kijanska, M., Kalie, E., Siergiejuk, E., Lee, S. S., Semplicio, G., Stoffel, I., Brezovich, A., Verma, M., Hansmann, I., Ammerer, G., Hofmann, K., Tooze, S., and Peter, M. (2012) Binding of the Atg1/ULK1 kinase to the ubiquitin-like protein Atg8 regulates autophagy. EMBO J. 31, 3691-3703

24. Kimura, S., Noda, T., and Yoshimori, T. (2007) Dissection of the autophagosome maturation process by a novel reporter protein, tandem fluorescent-tagged LC3. Autophagy. 3, 452-460

25. Zhao, Y. G., and Zhang, H. (2019) Autophagosome maturation: An epic journey from the ER to lysosomes. Journal of Cell Biology. 218, 757-770

26. Galluzzi, L., and Green, D. R. (2019) Autophagy-Independent Functions of the Autophagy Machinery. Cell. 177, 1682-1699

27. Calì, T., Galli, C., Olivari, S., and Molinari, M. (2008) Segregation and rapid turnover of EDEM1 by an autophagy-like mechanism modulates standard ERAD and folding activities. Biochem. Biophys. Res. Commun. 371, 405-410

28. Kar, R., Singha, P. K., Venkatachalam, M. A., and Saikumar, P. (2009) A Novel Role for MAP1 LC3 in Non-Autophagic Cytoplasmic Vacuolation Death of Cancer Cells. Oncogene. 28, 25562568

29. Delorme-Axford, E., and Klionsky, D. J. (2020) The LC3-conjugation machinery specifies cargo loading and secretion of extracellular vesicles. Autophagy. 16, 1169-1171

30. DeSelm, C. J., Miller, B. C., Zou, W., Beatty, W. L., van Meel, E., Takahata, Y., Klumperman, J., Tooze, S. A., Teitelbaum, S. L., and Virgin, H. W. (2011) Autophagy proteins regulate the secretory component of osteoclastic bone resorption. Dev Cell. 21, 966-974

31. Leidal, A. M., Huang, H. H., Marsh, T., Solvik, T., Zhang, D., Ye, J., Kai, F., Goldsmith, J., Liu, J. Y., Huang, Y.-H., Monkkonen, T., Vlahakis, A., Huang, E. J., Goodarzi, H., Yu, L., Wiita, A. P., and Debnath, J. (2020) The LC3-conjugation machinery specifies the loading of RNA-binding proteins into extracellular vesicles. Nature Cell Biology. 22, 187-199

32. Nollet, M., Santucci-Darmanin, S., Breuil, V., Al-Sahlanee, R., Cros, C., Topi, M., Momier, D., Samson, M., Pagnotta, S., Cailleteau, L., Battaglia, S., Farlay, D., Dacquin, R., Barois, N., Jurdic, P., Boivin, G., Heymann, D., Lafont, F., Lu, S. S., Dempster, D. W., Carle, G. F., and PierrefiteCarle, V. (2014) Autophagy in osteoblasts is involved in mineralization and bone homeostasis. Autophagy. 10, 1965-1977

33. Patel, K. K., Miyoshi, H., Beatty, W. L., Head, R. D., Malvin, N. P., Cadwell, K., Guan, J.-L., Saitoh, T., Akira, S., Seglen, P. O., Dinauer, M. C., Virgin, H. W., and Stappenbeck, T. S. (2013) Autophagy proteins control goblet cell function by potentiating reactive oxygen species production. The EMBO Journal. 32, 3130-3144

34. Ponpuak, M., Mandell, M. A., Kimura, T., Chauhan, S., Cleyrat, C., and Deretic, V. (2015) Secretory autophagy. Curr Opin Cell Biol. 35, 106-116

35. Martinez, J. (2018) LAP it up, fuzz ball: A short history of LC3-associated phagocytosis. Curr Opin Immunol. 55, 54-61

36. Sandilands, E., Serrels, B., McEwan, D. G., Morton, J. P., Macagno, J. P., McLeod, K., Stevens, C., Brunton, V. G., Langdon, W. Y., Vidal, M., Sansom, O. J., Dikic, I., Wilkinson, S., and Frame, M. C. (2011) Autophagic targeting of Src promotes cancer cell survival following reduced FAK signalling. Nat. Cell Biol. 14, 51-60

37. Martinez-Lopez, N., Athonvarangkul, D., Mishall, P., Sahu, S., and Singh, R. (2013) Autophagy proteins regulate ERK phosphorylation. Nat Commun. 4, 2799 
38. Barrow-McGee, R., Kishi, N., Joffre, C., Ménard, L., Hervieu, A., Bakhouche, B. A., Noval, A. J., Mai, A., Guzmán, C., Robbez-Masson, L., Iturrioz, X., Hulit, J., Brennan, C. H., Hart, I. R., Parker, P. J., Ivaska, J., and Kermorgant, S. (2016) Beta 1-integrin-c-Met cooperation reveals an insidein survival signalling on autophagy-related endomembranes. Nat Commun. 7, 11942

39. Hayashi, K., Taura, M., and Iwasaki, A. (2018) The interaction between IKK $\alpha$ and LC3 promotes type I interferon production through the TLR9-containing LAPosome. Sci Signal. $10.1126 /$ scisignal.aan4144

40. Wong, J., Zhang, J., Si, X., Gao, G., Mao, I., McManus, B. M., and Luo, H. (2008) Autophagosome Supports Coxsackievirus B3 Replication in Host Cells. J Virol. 82, 9143-9153

41. Al-Younes, H. M., Al-Zeer, M. A., Khalil, H., Gussmann, J., Karlas, A., Machuy, N., Brinkmann, V., Braun, P. R., and Meyer, T. F. (2011) Autophagy-independent function of MAP-LC3 during intracellular propagation of Chlamydia trachomatis. Autophagy. 7, 814-828

42. Sin, J., McIntyre, L., Stotland, A., Feuer, R., and Gottlieb, R. A. (2017) Coxsackievirus B Escapes the Infected Cell in Ejected Mitophagosomes. Journal of Virology. 10.1128/JVI.01347-17

43. Selleck, E. M., Orchard, R. C., Lassen, K. G., Beatty, W. L., Xavier, R. J., Levine, B., Virgin, H. W., and Sibley, L. D. (2015) A Noncanonical Autophagy Pathway Restricts Toxoplasma gondii Growth in a Strain-Specific Manner in IFN- $\gamma$-Activated Human Cells. mBio. 10.1128/mBio.01157-15

44. Kraft, L. J., Nguyen, T. A., Vogel, S. S., and Kenworthy, A. K. (2014) Size, stoichiometry, and organization of soluble LC3-associated complexes. Autophagy. 10, 861-877

45. Kraft, L. J., Manral, P., Dowler, J., and Kenworthy, A. K. (2016) Nuclear LC3 associates with slowly diffusing complexes that survey the nucleolus. Traffic. 17, 369-399

46. Kraft, L. J., Dowler, J., Manral, P., and Kenworthy, A. K. (2016) Size, organization, and dynamics of soluble SQSTM1 and LC3-SQSTM1 complexes in living cells. Autophagy. 12, 1660-1674

47. Agrotis, A., von Chamier, L., Oliver, H., Kiso, K., Singh, T., and Ketteler, R. (2019) Human ATG4 autophagy proteases counteract attachment of ubiquitin-like LC3/GABARAP proteins to other cellular proteins. J. Biol. Chem. 294, 12610-12621

48. Hung, V., Udeshi, N. D., Lam, S. S., Loh, K. H., Cox, K. J., Pedram, K., Carr, S. A., and Ting, A. Y. (2016) Spatially resolved proteomic mapping in living cells with the engineered peroxidase APEX2. Nat Protoc. 11, 456-475

49. Kostelnik, K. B., Barker, A., Schultz, C., Mitchell, T. P., Rajeeve, V., White, I. J., Aurrand-Lions, M., Nourshargh, S., Cutillas, P., and Nightingale, T. D. (2019) Dynamic trafficking and turnover of JAM-C is essential for endothelial cell migration. PLoS Biol. 17, e3000554

50. Rhee, H.-W., Zou, P., Udeshi, N. D., Martell, J. D., Mootha, V. K., Carr, S. A., and Ting, A. Y. (2013) Proteomic mapping of mitochondria in living cells via spatially restricted enzymatic tagging. Science. 339, 1328-1331

51. Yamamoto, A., Tagawa, Y., Yoshimori, T., Moriyama, Y., Masaki, R., and Tashiro, Y. (1998) Bafilomycin A1 prevents maturation of autophagic vacuoles by inhibiting fusion between autophagosomes and lysosomes in rat hepatoma cell line, H-4-II-E cells. Cell Struct. Funct. 23, 33-42

52. Thoreen, C. C., Kang, S. A., Chang, J. W., Liu, Q., Zhang, J., Gao, Y., Reichling, L. J., Sim, T., Sabatini, D. M., and Gray, N. S. (2009) An ATP-competitive mammalian target of rapamycin inhibitor reveals rapamycin-resistant functions of mTORC1. J. Biol. Chem. 284, 8023-8032

53. Martell, J. D., Deerinck, T. J., Sancak, Y., Poulos, T. L., Mootha, V. K., Sosinsky, G. E., Ellisman, M. H., and Ting, A. Y. (2012) Engineered ascorbate peroxidase as a genetically encoded reporter for electron microscopy. Nat. Biotechnol. 30, 1143-1148

54. Huang, D. W., Sherman, B. T., and Lempicki, R. A. (2009) Bioinformatics enrichment tools: paths toward the comprehensive functional analysis of large gene lists. Nucleic Acids Res. 37, $1-13$ 
55. Huang, D. W., Sherman, B. T., and Lempicki, R. A. (2009) Systematic and integrative analysis of large gene lists using DAVID bioinformatics resources. Nat Protoc. 4, 44-57

56. Consortium UniProt (2019) UniProt: a worldwide hub of protein knowledge. Nucleic Acids Res. 47, D506-D515

57. Dengjel, J., Høyer-Hansen, M., Nielsen, M. O., Eisenberg, T., Harder, L. M., Schandorff, S., Farkas, T., Kirkegaard, T., Becker, A. C., Schroeder, S., Vanselow, K., Lundberg, E., Nielsen, M. M., Kristensen, A. R., Akimov, V., Bunkenborg, J., Madeo, F., Jäättelä, M., and Andersen, J. S. (2012) Identification of autophagosome-associated proteins and regulators by quantitative proteomic analysis and genetic screens. Mol Cell Proteomics. 11, M111.014035

58. Mancias, J. D., Wang, X., Gygi, S. P., Harper, J. W., and Kimmelman, A. C. (2014) Quantitative proteomics identifies NCOA4 as the cargo receptor mediating ferritinophagy. Nature. 509, 105-109

59. Gao, W., Kang, J. H., Liao, Y., Ding, W.-X., Gambotto, A. A., Watkins, S. C., Liu, Y.-J., Stolz, D. B., and Yin, X.-M. (2010) Biochemical isolation and characterization of the tubulovesicular LC3positive autophagosomal compartment. J Biol Chem. 285, 1371-1383

60. Le Guerroué, F., Eck, F., Jung, J., Starzetz, T., Mittelbronn, M., Kaulich, M., and Behrends, C. (2017) Autophagosomal Content Profiling Reveals an LC3C-Dependent Piecemeal Mitophagy Pathway. Mol Cell. 68, 786-796.e6

61. Kalvari, I., Tsompanis, S., Mulakkal, N. C., Osgood, R., Johansen, T., Nezis, I. P., and Promponas, V. J. (2014) ilIR. Autophagy. 10, 913-925

62. Gao, W., Chen, Z., Wang, W., and Stang, M. T. (2013) E1-Like Activating Enzyme Atg7 Is Preferentially Sequestered into p62 Aggregates via Its Interaction with LC3-I. PLoS One. 10.1371/journal.pone.0073229

63. Huang, R., and Liu, W. (2015) Identifying an essential role of nuclear LC3 for autophagy. Autophagy. 11, 852-853

64. Verlhac, P., Grégoire, I. P., Azocar, O., Petkova, D. S., Baguet, J., Viret, C., and Faure, M. (2015) Autophagy Receptor NDP52 Regulates Pathogen-Containing Autophagosome Maturation. Cell Host \& Microbe. 17, 515-525

65. Kim, J., Kundu, M., Viollet, B., and Guan, K.-L. (2011) AMPK and mTOR regulate autophagy through direct phosphorylation of Ulk1. Nat. Cell Biol. 13, 132-141

66. Lippai, M., and Lőw, P. (2014) The role of the selective adaptor p62 and ubiquitin-like proteins in autophagy. Biomed Res Int. 2014, 832704

67. Deng, Z., Purtell, K., Lachance, V., Wold, M. S., Chen, S., and Yue, Z. (2017) Autophagy Receptors and Neurodegenerative Diseases. Trends in Cell Biology. 27, 491-504

68. Bachar-Wikstrom, E., Wikstrom, J. D., Ariav, Y., Tirosh, B., Kaiser, N., Cerasi, E., and Leibowitz, G. (2013) Stimulation of Autophagy Improves Endoplasmic Reticulum Stress-Induced Diabetes. Diabetes. 62, 1227-1237

69. Gong, C., and Xia, H. (2020) Resveratrol suppresses melanoma growth by promoting autophagy through inhibiting the PI3K/AKT/mTOR signaling pathway. Exp Ther Med. 19, 1878-1886

70. Zhang, W., Zhou, M., Lu, W., Gong, J., Gao, F., Li, Y., Xu, X., Lin, Y., Zhang, X., Ding, L., Zhang, Z., Li, G., Chen, X., Sun, X., Zhu, X., Xu, P., and Zhang, Y. (2020) CNTNAP4 deficiency in dopaminergic neurons initiates parkinsonian phenotypes. Theranostics. 10, 3000-3021

71. He, J., Vora, M., Haney, R. M., Filonov, G. S., Musselman, C. A., Burd, C. G., Kutateladze, A. G., Verkhusha, V. V., Stahelin, R. V., and Kutateladze, T. G. (2009) Membrane insertion of the FYVE domain is modulated by $\mathrm{pH}$. Proteins. 76, 852-860

72. Patki, V., Virbasius, J., Lane, W. S., Toh, B. H., Shpetner, H. S., and Corvera, S. (1997) Identification of an early endosomal protein regulated by phosphatidylinositol 3-kinase. Proc. Natl. Acad. Sci. U.S.A. 94, 7326-7330 
73. Simonsen, A., Lippé, R., Christoforidis, S., Gaullier, J. M., Brech, A., Callaghan, J., Toh, B. H., Murphy, C., Zerial, M., and Stenmark, H. (1998) EEA1 links PI(3)K function to Rab5 regulation of endosome fusion. Nature. 394, 494-498

74. Handbook of Cell Signaling (2010) Handbook of Cell Signaling, Elsevier, 10.1016/B978-0-12374145-5.X0001-0

75. Wu, X., Fleming, A., Ricketts, T., Pavel, M., Virgin, H., Menzies, F. M., and Rubinsztein, D. C. (2016) Autophagy regulates Notch degradation and modulates stem cell development and neurogenesis. Nature Communications. 7, 10533

76. Razi, M., Chan, E. Y. W., and Tooze, S. A. (2009) Early endosomes and endosomal coatomer are required for autophagy. $J$ Cell Biol. 185, 305-321

77. Hunker, C. M., Galvis, A., Kruk, I., Giambini, H., Veisaga, M. L., and Barbieri, M. A. (2006) Rab5activating protein 6 , a novel endosomal protein with a role in endocytosis. Biochem. Biophys. Res. Commun. 340, 967-975

78. Jackson, R. J., Hellen, C. U. T., and Pestova, T. V. (2010) The mechanism of eukaryotic translation initiation and principles of its regulation. Nat Rev Mol Cell Biol. 11, 113-127

79. Humeau, J., Sauvat, A., Cerrato, G., Xie, W., Loos, F., Iannantuoni, F., Bezu, L., Lévesque, S., Paillet, J., Pol, J., Leduc, M., Zitvogel, L., de Thé, H., Kepp, O., and Kroemer, G. (2020) Inhibition of transcription by dactinomycin reveals a new characteristic of immunogenic cell stress. EMBO Mol Med. 10.15252/emmm.201911622

80. Bretin et al., A. (2016) Activation of the EIF2AK4-EIF2A/elF2 $\alpha$-ATF4 pathway triggers autophagy response to Crohn disease-associated adherent-invasive Escherichia coli infection

81. Amano, M., Nakayama, M., and Kaibuchi, K. (2010) Rho-Kinase/ROCK: A Key Regulator of the Cytoskeleton and Cell Polarity. Cytoskeleton (Hoboken). 67, 545-554

82. Cudjoe, E. K., Saleh, T., Hawkridge, A. M., and Gewirtz, D. A. (2017) Proteomics Insights into Autophagy. Proteomics. 17, 1700022

83. Mijaljica, D., Prescott, M., and Devenish, R. J. (2011) Microautophagy in mammalian cells: revisiting a 40-year-old conundrum. Autophagy. 7, 673-682

84. Juste, Y. R., and Cuervo, A. M. (2019) Analysis of Chaperone-Mediated Autophagy. in Autophagy: Methods and Protocols (Ktistakis, N., and Florey, O. eds), pp. 703-727, Methods in Molecular Biology, Springer, New York, NY, 10.1007/978-1-4939-8873-0_47

85. Codogno, P., Mehrpour, M., and Proikas-Cezanne, T. (2012) Canonical and non-canonical autophagy: variations on a common theme of self-eating? Nat Rev Mol Cell Biol. 13, 7-12

86. Fletcher, K., Ulferts, R., Jacquin, E., Veith, T., Gammoh, N., Arasteh, J. M., Mayer, U., Carding, S. R., Wileman, T., Beale, R., and Florey, O. (2018) The WD40 domain of ATG16L1 is required for its non-canonical role in lipidation of LC3 at single membranes. EMBO J.

10.15252/embj.201797840

87. Mauvezin, C., Sancho, A., Ivanova, S., Palacin, M., and Zorzano, A. (2012) DOR undergoes nucleo-cytoplasmic shuttling, which involves passage through the nucleolus. FEBS Lett. 586, 3179-3186

88. Mauvezin, C., Orpinell, M., Francis, V. A., Mansilla, F., Duran, J., Ribas, V., Palacín, M., Boya, P., Teleman, A. A., and Zorzano, A. (2010) The nuclear cofactor DOR regulates autophagy in mammalian and Drosophila cells. EMBO Rep. 11, 37-44

89. Huang, R., Xu, Y., Wan, W., Shou, X., Qian, J., You, Z., Liu, B., Chang, C., Zhou, T., LippincottSchwartz, J., and Liu, W. (2015) Deacetylation of nuclear LC3 drives autophagy initiation under starvation. Mol Cell. 57, 456-466

90. Shim, M. S., Nettesheim, A., Hirt, J., and Liton, P. B. (2020) The autophagic protein LC3 translocates to the nucleus and localizes in the nucleolus associated to NUFIP1 in response to cyclic mechanical stress. Autophagy. 16, 1248-1261

91. Sjöblom, B., Salmazo, A., and Djinović-Carugo, K. (2008) Alpha-actinin structure and regulation. Cell. Mol. Life Sci. 65, 2688-2701 
92. Yee, A., Papillon, J., Guillemette, J., Kaufman, D. R., Kennedy, C. R. J., and Cybulsky, A. V. (2018) Proteostasis as a therapeutic target in glomerular injury associated with mutant $\alpha$ actinin-4. American Journal of Physiology-Renal Physiology. 315, F954-F966

93. Lam, S. S., Martell, J. D., Kamer, K. J., Deerinck, T. J., Ellisman, M. H., Mootha, V. K., and Ting, A. Y. (2015) Directed evolution of APEX2 for electron microscopy and proximity labeling. Nat. Methods. 12, 51-54

94. Agrotis, A., Pengo, N., Burden, J. J., and Ketteler, R. (2019) Redundancy of human ATG4 protease isoforms in autophagy and LC3/GABARAP processing revealed in cells. Autophagy. 10.1080/15548627.2019.1569925

95. Rajeeve, V., Vendrell, I., Wilkes, E., Torbett, N., and Cutillas, P. R. (2014) Cross-species Proteomics Reveals Specific Modulation of Signaling in Cancer and Stromal Cells by Phosphoinositide 3-kinase (PI3K) Inhibitors. Mol Cell Proteomics. 13, 1457-1470

96. Hijazi, M., Smith, R., Rajeeve, V., Bessant, C., and Cutillas, P. R. (2020) Reconstructing kinase network topologies from phosphoproteomics data reveals cancer-associated rewiring. Nat Biotechnol. 38, 493-502

97. Perez-Riverol, Y., Csordas, A., Bai, J., Bernal-Llinares, M., Hewapathirana, S., Kundu, D. J., Inuganti, A., Griss, J., Mayer, G., Eisenacher, M., Pérez, E., Uszkoreit, J., Pfeuffer, J., Sachsenberg, T., Yilmaz, S., Tiwary, S., Cox, J., Audain, E., Walzer, M., Jarnuczak, A. F., Ternent, T., Brazma, A., and Vizcaíno, J. A. (2019) The PRIDE database and related tools and resources in 2019: improving support for quantification data. Nucleic Acids Res. 47, D442-D450

98. R Core Team (2020) R Core Team (2020). R: A language and environment for statistical computing. R Foundation for Statistical Computing, Vienna, Austria. [online] https://www.eea.europa.eu/data-and-maps/indicators/oxygen-consuming-substances-inrivers/r-development-core-team-2006 (Accessed January 16, 2021)

99. Benjamini, Y., and Hochberg, Y. (1995) Controlling the False Discovery Rate: A Practical and Powerful Approach to Multiple Testing. Journal of the Royal Statistical Society. Series B (Methodological). 57, 289-300

100. Wickham, H. (2016) ggplot2: Elegant Graphics for Data Analysis, 2nd Ed., Use R!, Springer International Publishing, 10.1007/978-3-319-24277-4

101. Wickham, H. (2007) Reshaping Data with the reshape Package. Journal of Statistical Software. 21, 1-20

102. Frank E. Harrell (2020) Hmisc: A package of miscellaneous $R$ functions., [online] https://CRAN.R-project.org/package=Hmisc (Accessed January 16, 2021)

103. Warnes (2013) gplots: Various R programming tools for plotting data - ScienceOpen. [online] https://www.scienceopen.com/document?vid=0e5d8e31-1fe4-492f-a3d8-8cd71b2b8ad9 (Accessed January 16, 2021)

104. Bryan, J., W., H. (2017) readxl: Read Excel Files. [online] https://readxl.tidyverse.org/ (Accessed January 16, 2021)

105. J. Irisson., S., K. (2016) Getting started with ggrepel. [online] https://cran.rproject.org/web/packages/ggrepel/vignettes/ggrepel.html (Accessed January 16, 2021)

106. Szklarczyk, D., Franceschini, A., Wyder, S., Forslund, K., Heller, D., Huerta-Cepas, J., Simonovic, M., Roth, A., Santos, A., Tsafou, K. P., Kuhn, M., Bork, P., Jensen, L. J., and von Mering, C. (2015) STRING v10: protein-protein interaction networks, integrated over the tree of life. Nucleic Acids Research. 43, D447-D452 


\section{Figure 1}

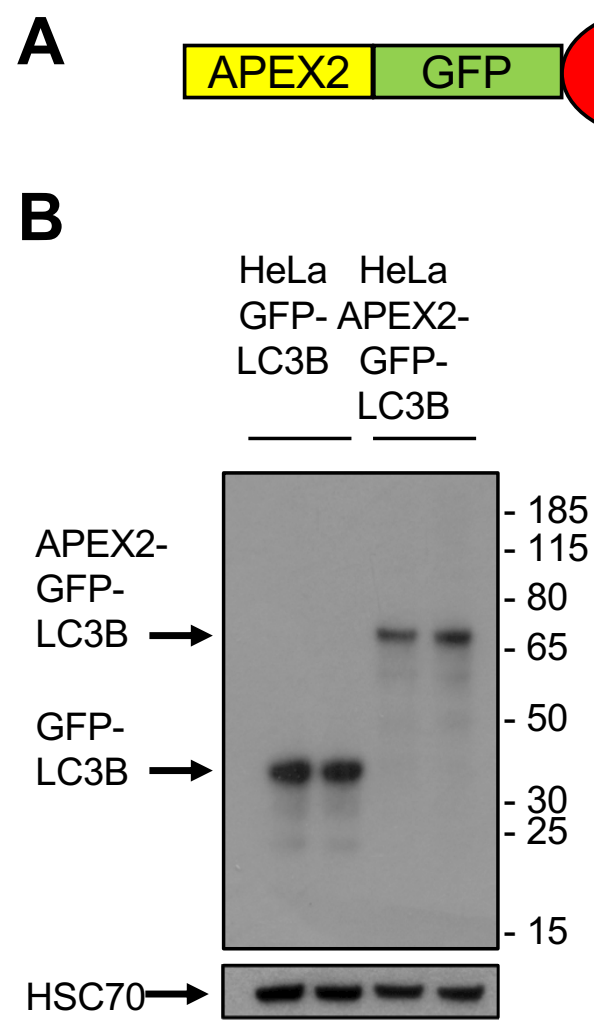

C
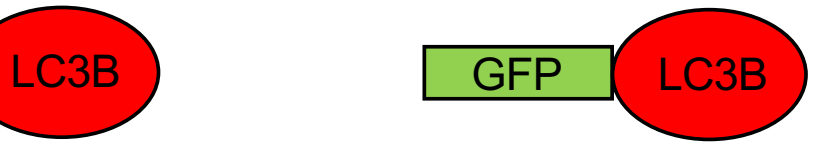

B

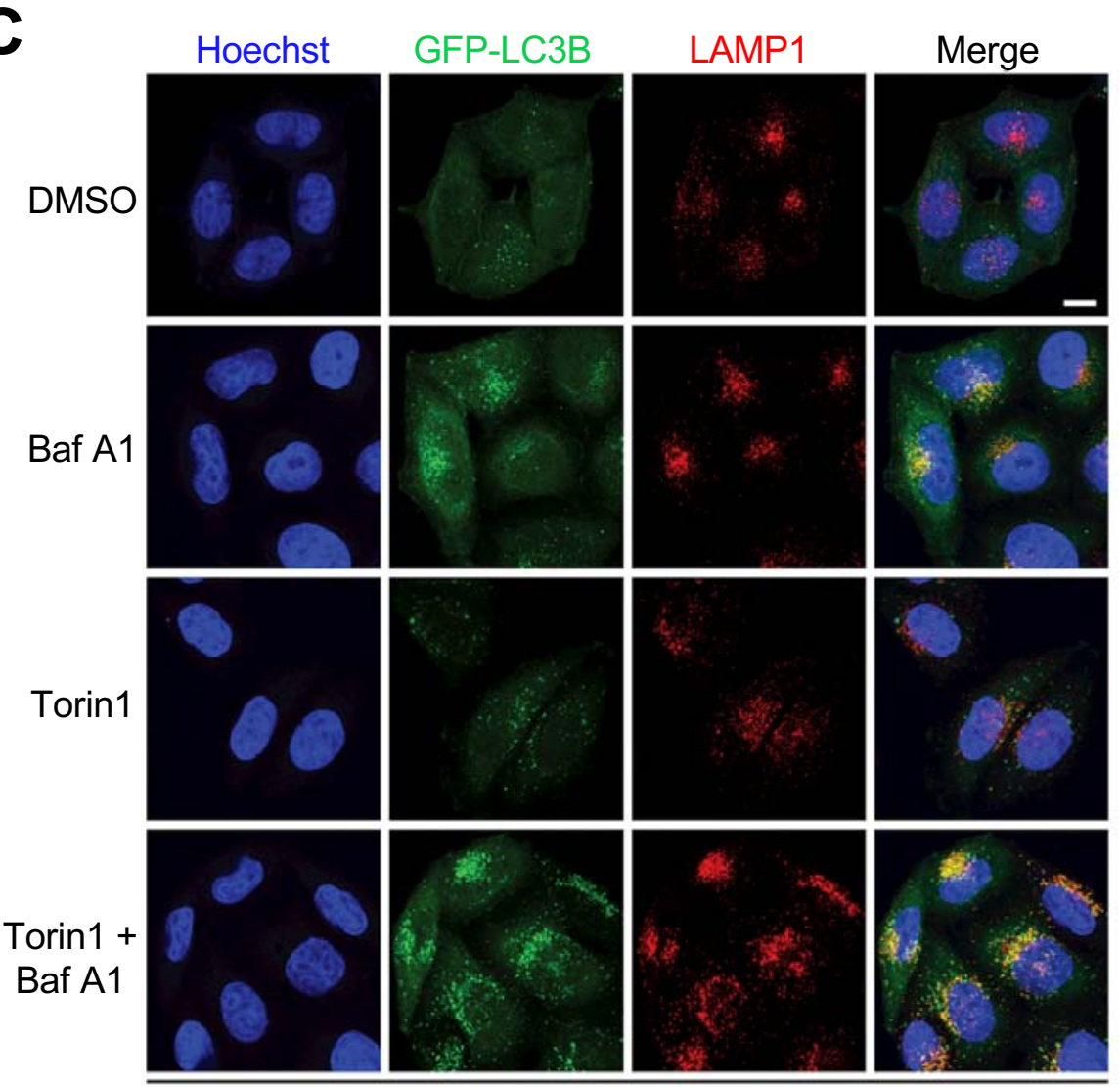

HeLa APEX2-GFP-LC3B

D

- DAB substrate

+ DAB substrate
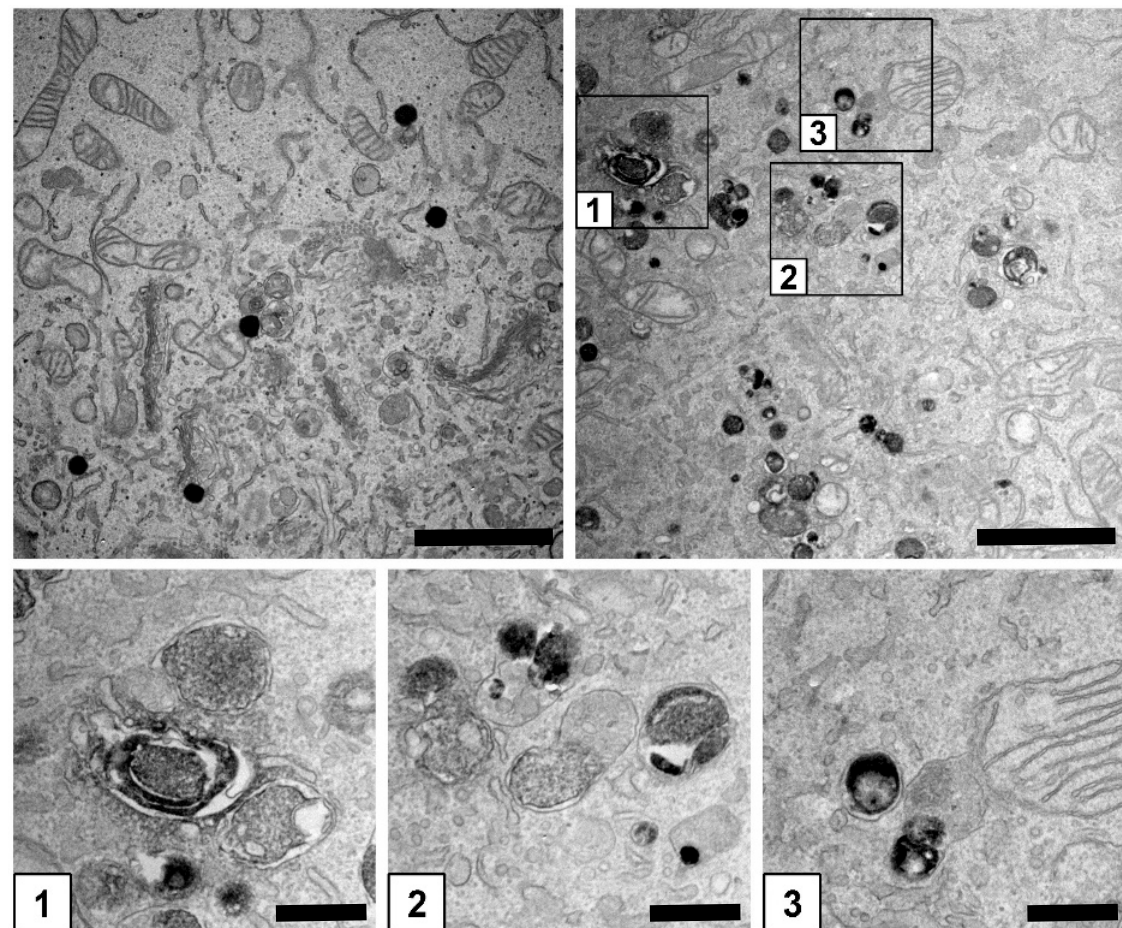

HeLa APEX2-GFP-LC3B 


\section{Figure 2}

A

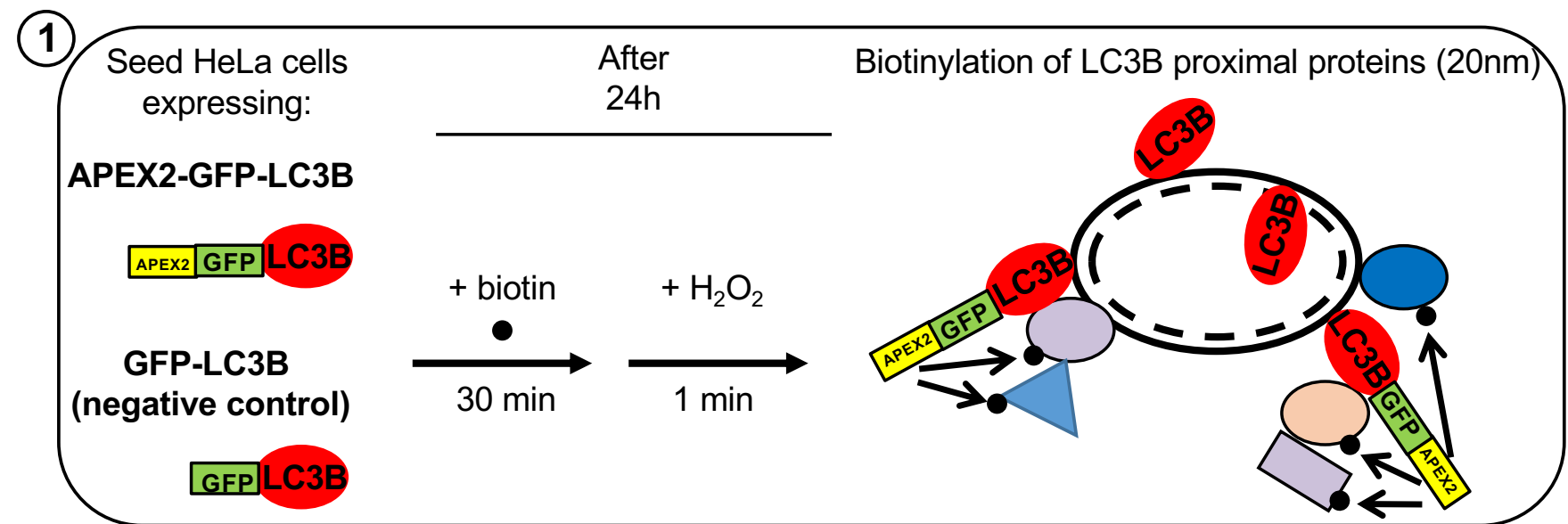

(2)

Technique validation

Fix and stain with streptavidin546

Confocal analysis

Lyse cells, neutravidin pull-down

Western blots

(Streptavidin and LC3B)

B

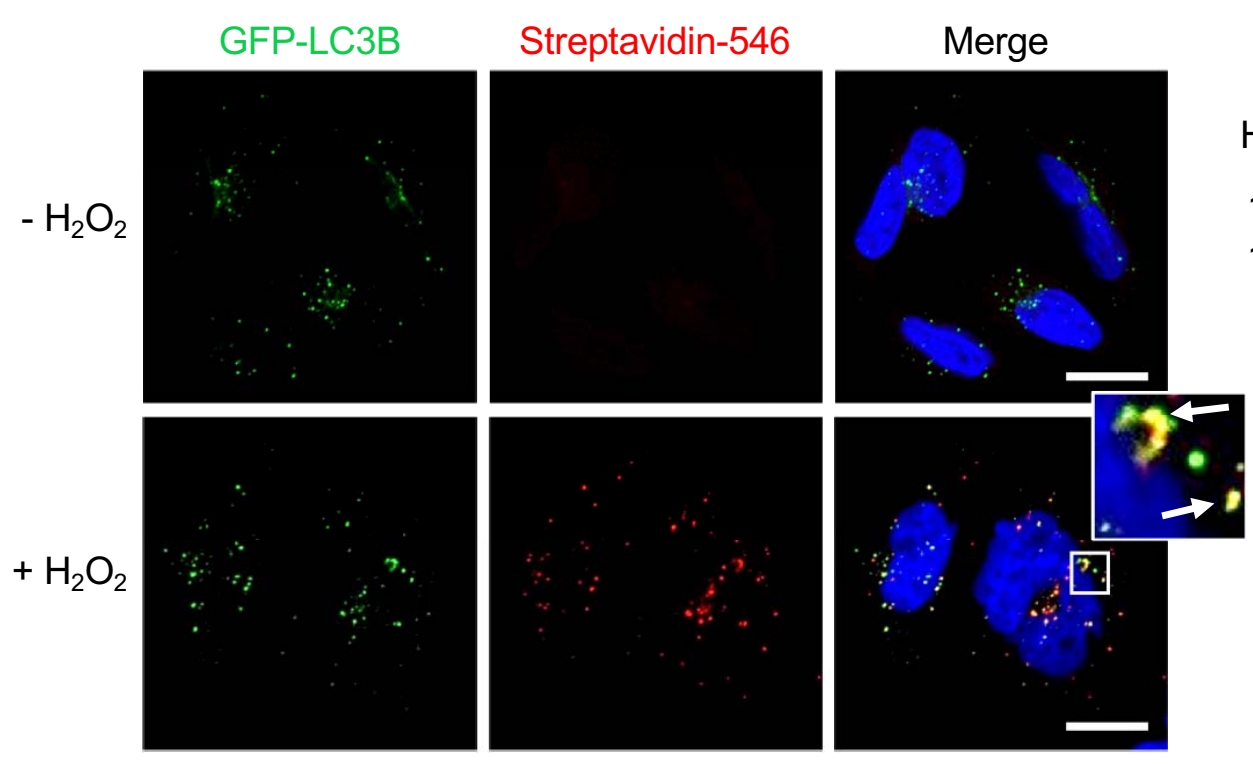

HeLa APEX2-GFP-LC3B
LC3B proximity interaction network

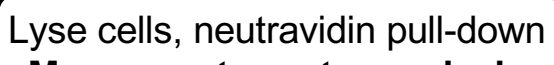

Mass spectrometry analysis

Determine hits following log $2>1$, MASCOT

score $>50,>2$ peptides identified and $p$-Value $<0.05$

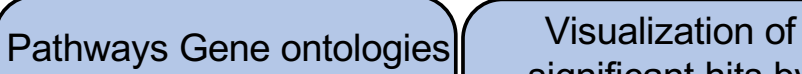
enrichment analysis:

- Cellular localisation

- Biological processes significant hits by volcano plot ( $p$-Value or

FDR score versus magnitude of change)
(4)

Hit validation

Comparison with previous studies

- Determination of putative / validated LIR domains

- Hit validation by coimmunoprecipitation and co-

immunofluorescence
C Neutravidin pull-down Lysates

Hela Hela Hela Hela GFP- APEX2- GFP- APEX2LC3B GFP-LC3B LC3B GFP-LC3B

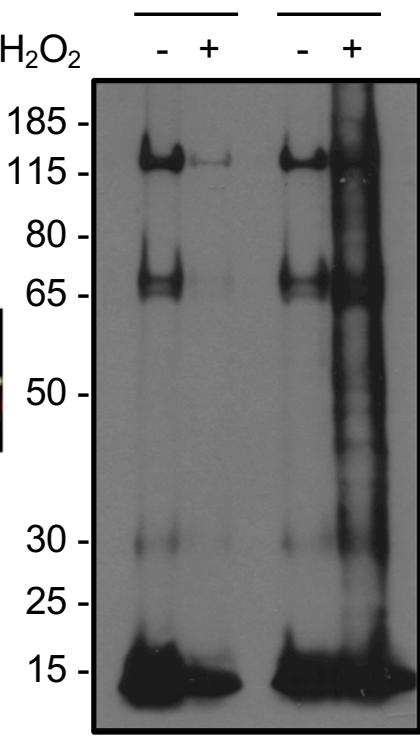

Streptavidin

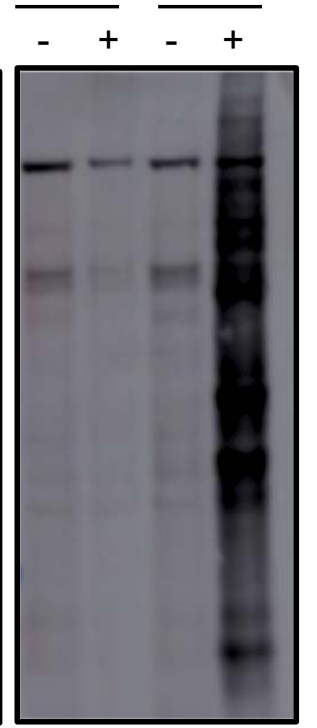

Streptavidin

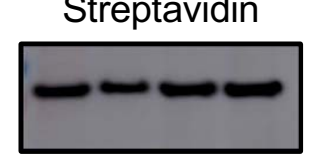

HSC70 


\section{Figure 3}

A Neutravidin pull-down

HeLa HeLa

GFP- APEX2-

LC3B GFP-LC3B

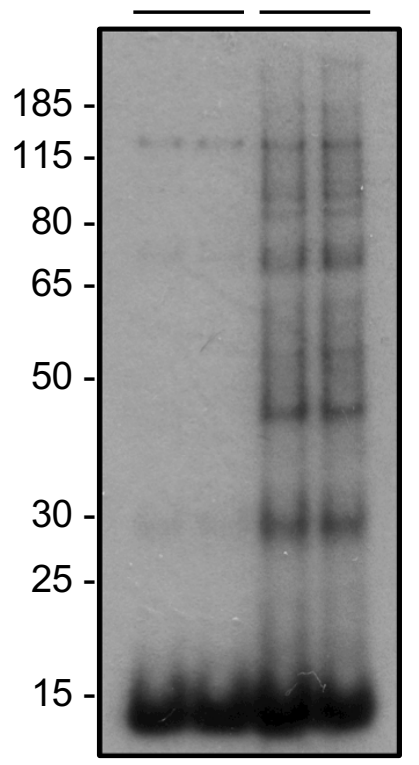

Streptavidin
B Neutravidin pull-down

HeLa HeLa

GFP- APEX2-

LC3B GFP-LC3B

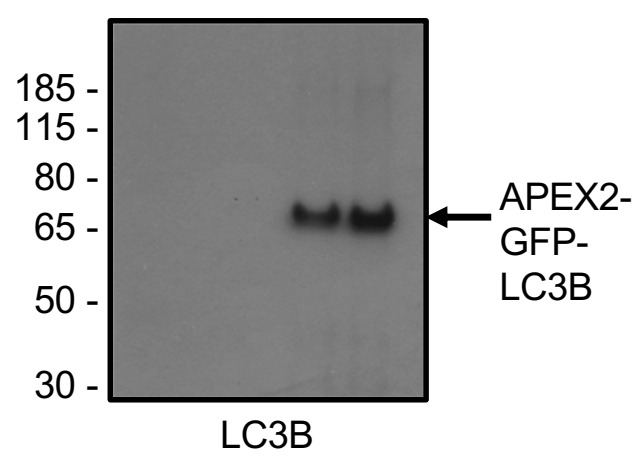

C Distribution of the 407 hits across the experiments

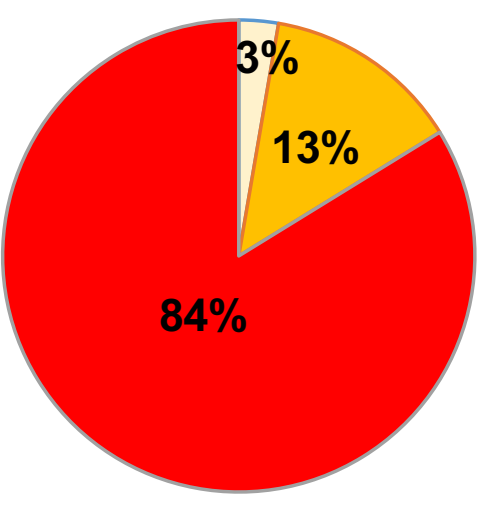

$\square 2$ experiments $\square 3$ experiments $\square 4$ experiments

D

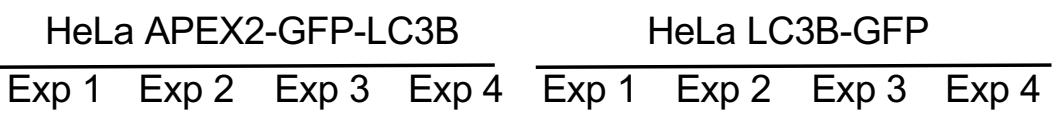
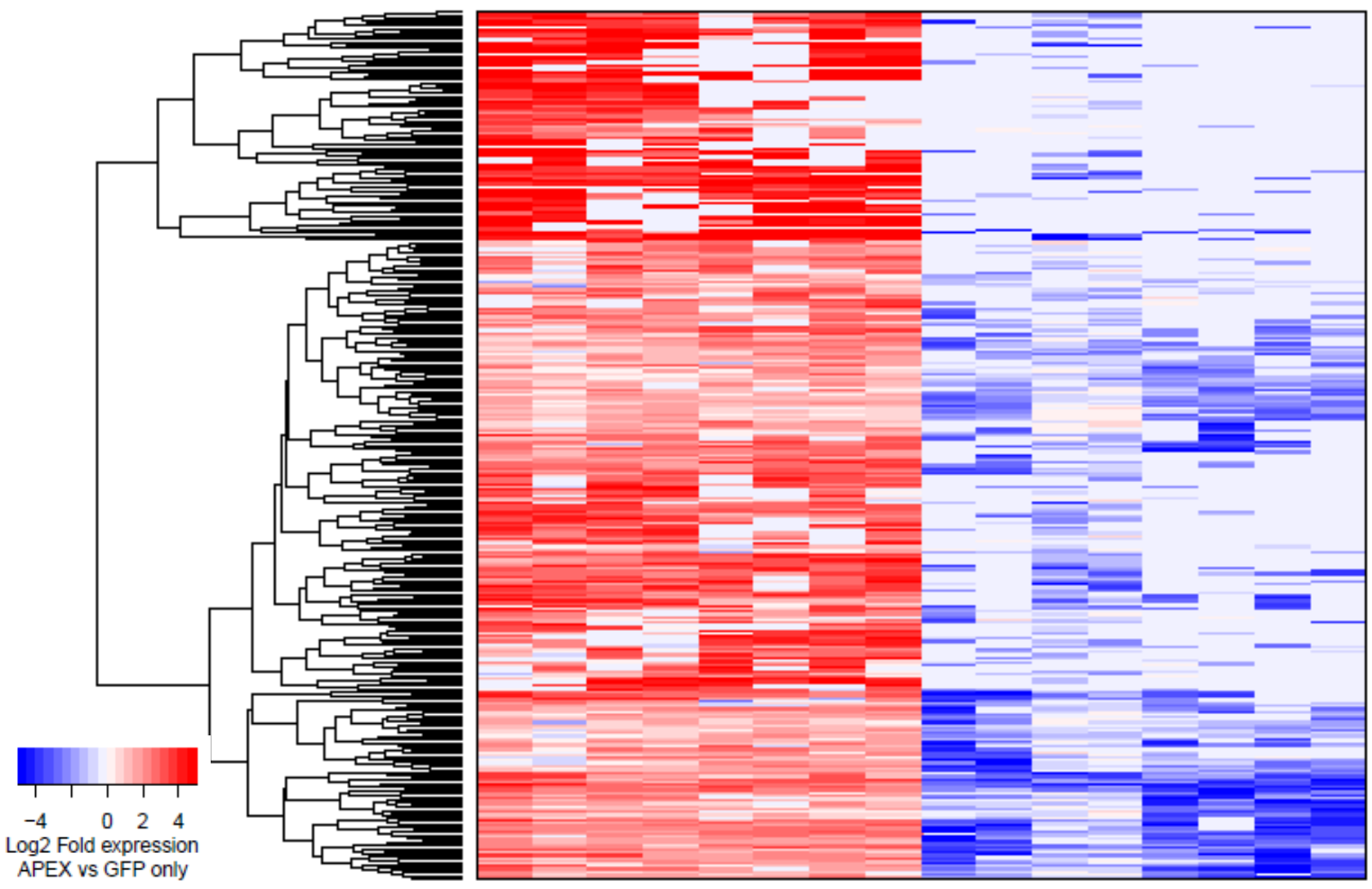

Total of 407 hits 


\section{Figure 4}

A

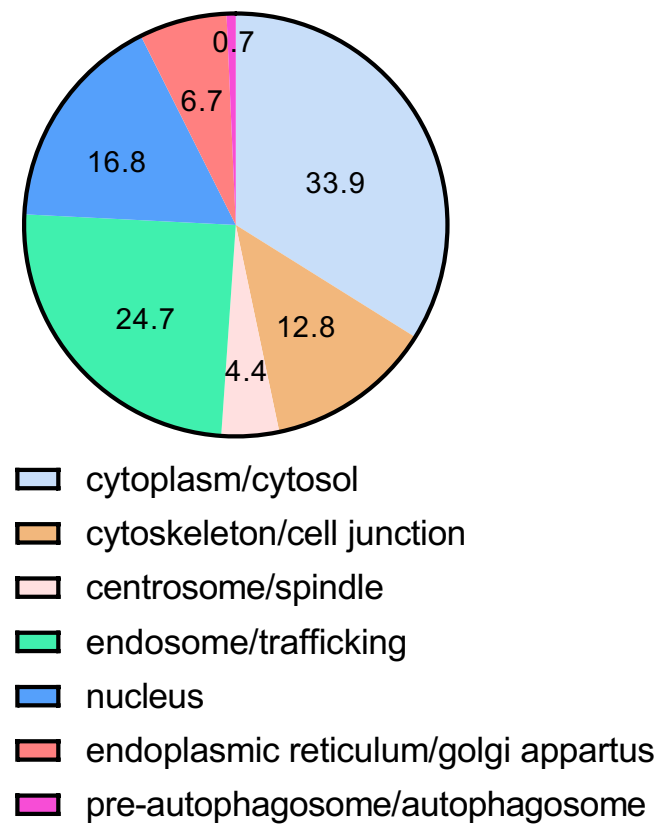

C

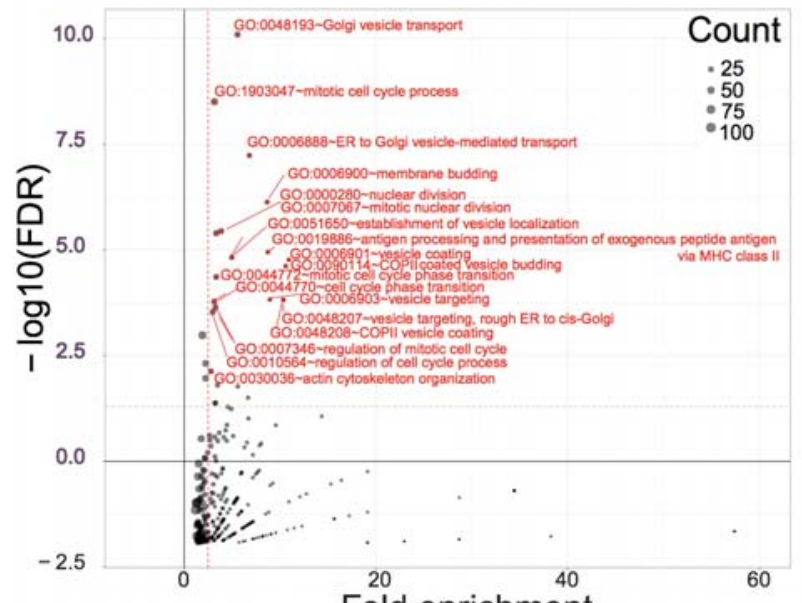

$\mathbf{D}$

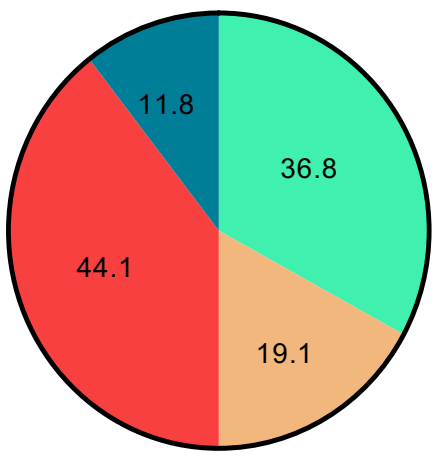
Membrane trafficking
Actin cytoskeleton organization
$\square$ Cell cycle/ mitosis

Antigen processing and presentation of exogenous peptide antigen via MHC class II

\section{B}
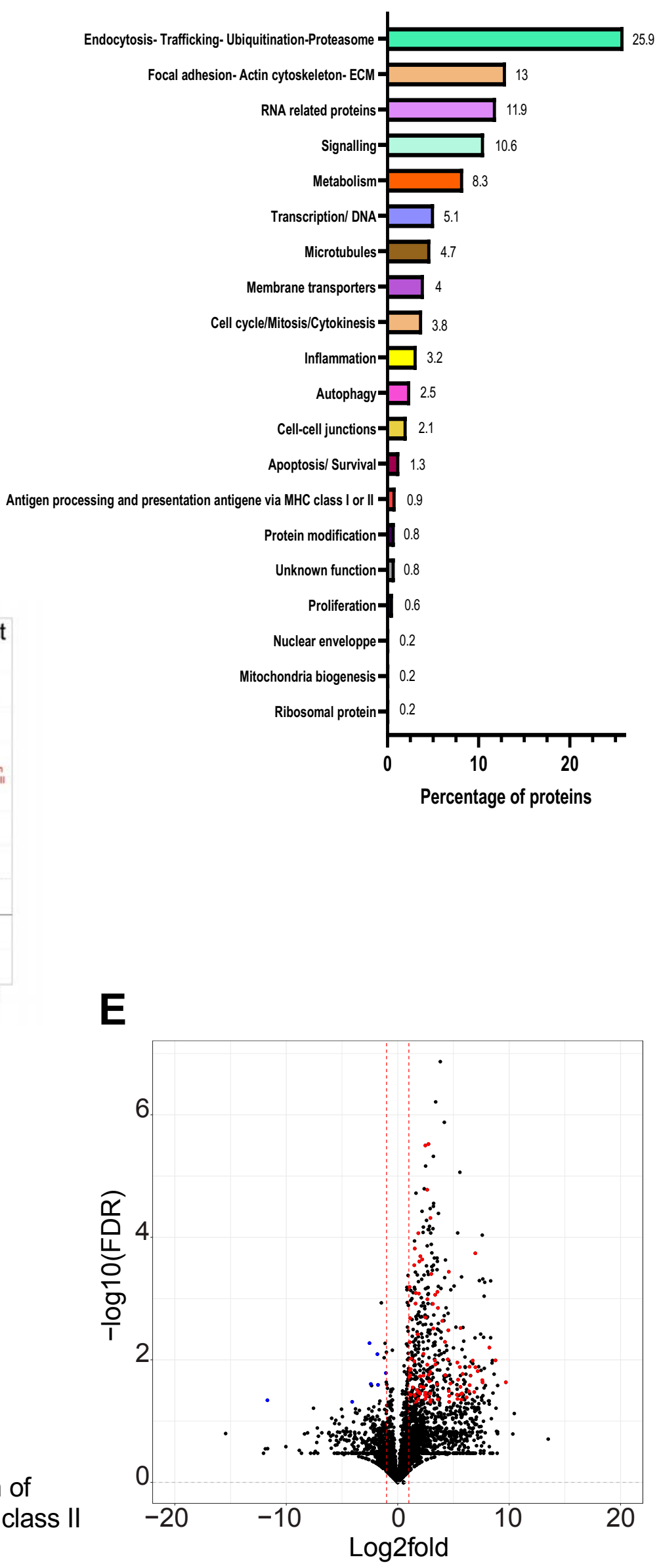


\section{Figure 5}

A

\section{1 autophagy-related hits,} 30 associated with LC3

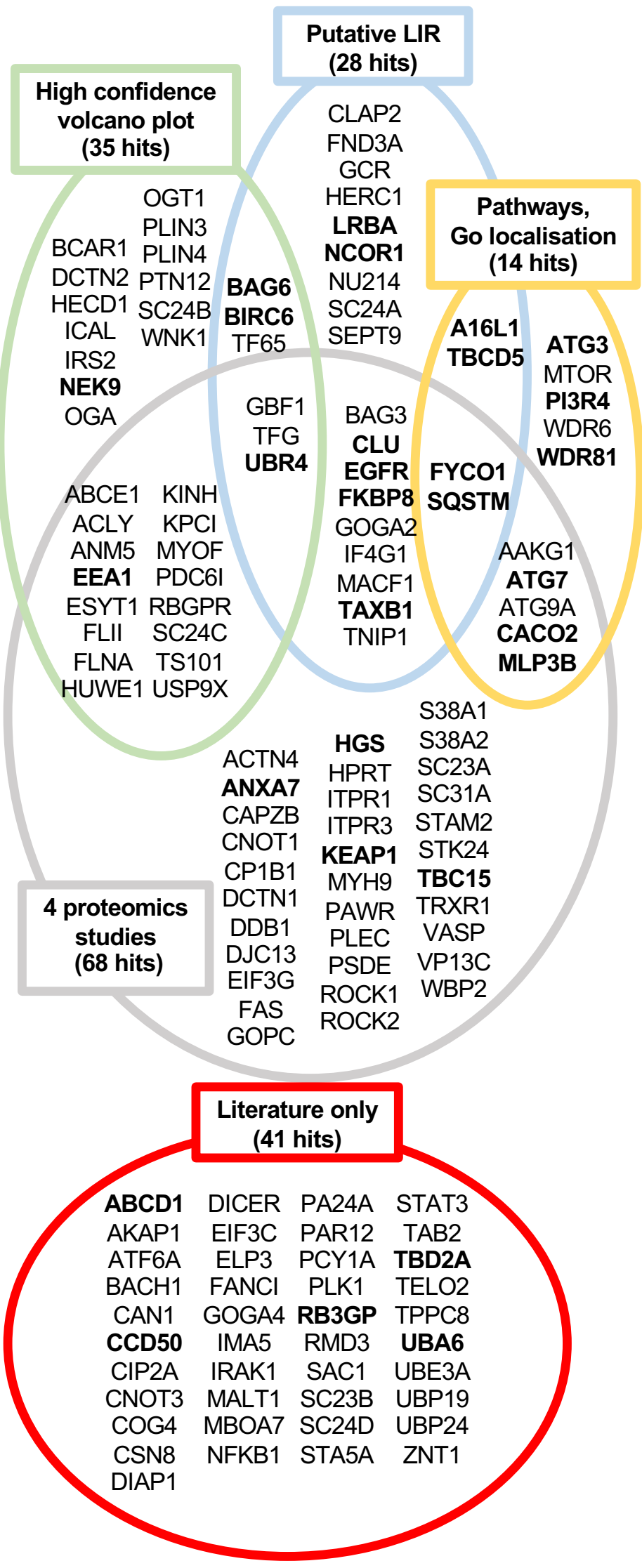

B
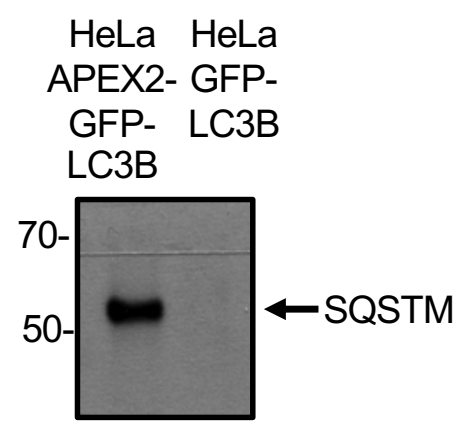

C
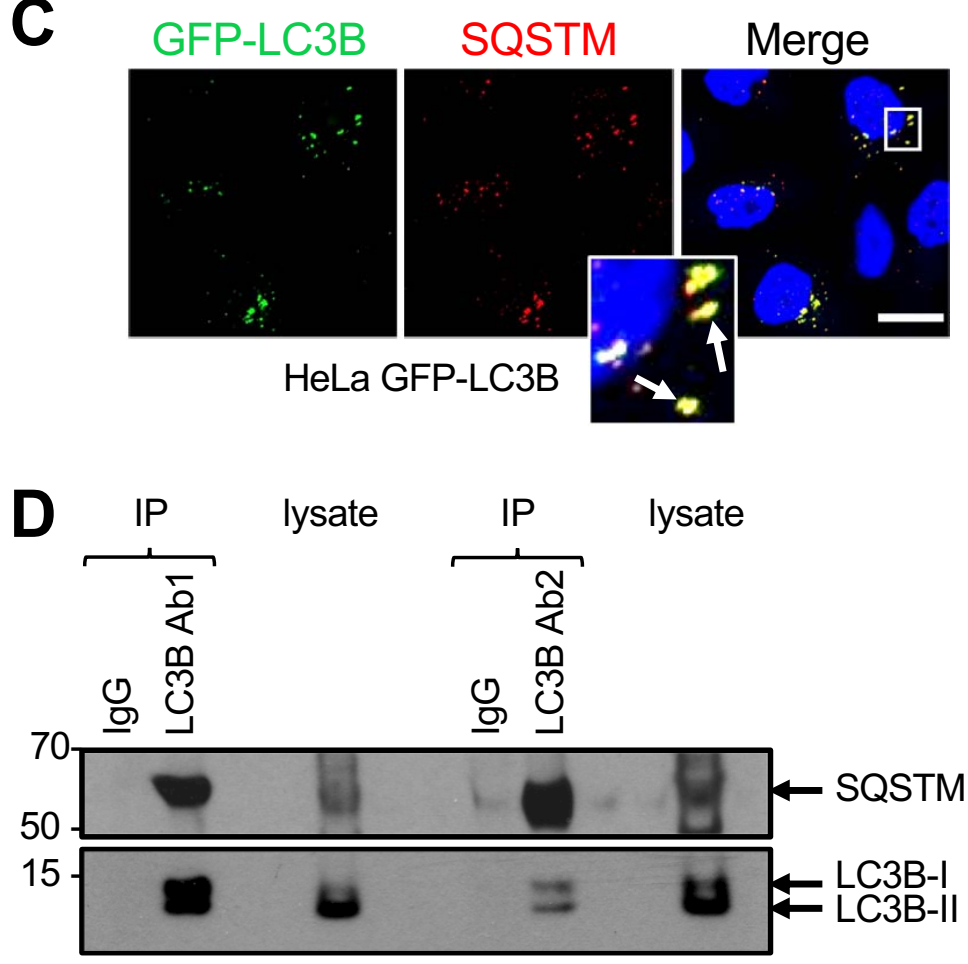

HeLa

E

HeLa HeLa

APEX2- GFP-

GFP- LC3B

LC3B

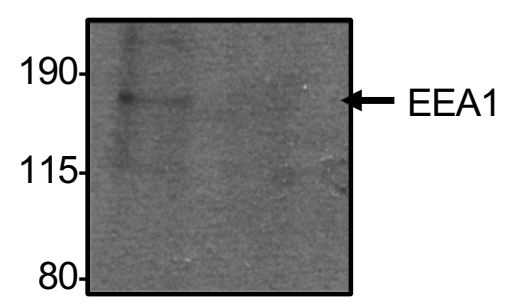

$\mathbf{F}$
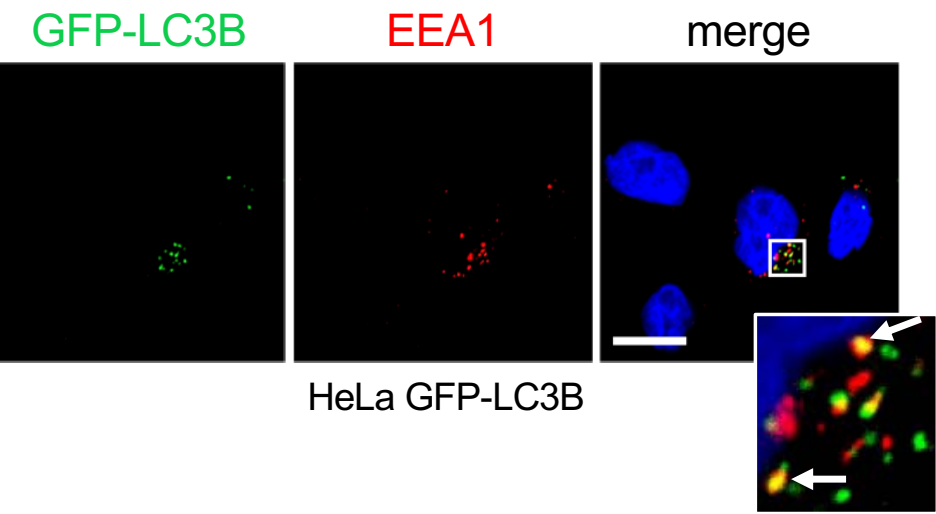


\section{Figure 6}

A

41 hits with
putative LIR and
not related to
autophagy

\begin{tabular}{|c|c|}
\cline { 2 - 2 } \multicolumn{1}{l|}{} & Log2fold \\
\hline AASD1 & 1.7 \\
\hline ACAP2 & 4.2 \\
\hline ANKL2 & 4.1 \\
\hline ANX11 & 1.6 \\
\hline BCAR3 & 7.9 \\
\hline BUB1B & 2.6 \\
\hline CNO10 & 3.3 \\
\hline CYTSB & 6 \\
\hline DLG1 & 5.4 \\
\hline DLGP5 & 4.6 \\
\hline DNMBP & 5.8 \\
\hline EIF2D & 2.1 \\
\hline EP15R & 2.9 \\
\hline EPIPL & 1.9 \\
\hline GAPD1 & 2.6 \\
\hline GOGA3 & 4.3 \\
\hline GOGB1 & 9.7 \\
\hline INF2 & 2.3 \\
\hline JIP4 & 3.2 \\
\hline KI13A & 6 \\
\hline KIF14 & 3.8 \\
\hline LIMC1 & 2.5 \\
\hline LR16A & 1.6 \\
\hline MIA3 & 4.7 \\
\hline OSBP1 & 3.4 \\
\hline PDE3A & 3.2 \\
\hline PJA2 & 4.7 \\
\hline PRC2C & 2.7 \\
\hline R3HD1 & 2.5 \\
\hline RABE1 & 7 \\
\hline RABE2 & 6.4 \\
\hline SH3G1 & 1.5 \\
\hline SPD2B & 2.7 \\
\hline SPG20 & 2.4 \\
\hline STRN & 3.4 \\
\hline TACC2 & 1.7 \\
\hline TENS3 & 3.2 \\
\hline TENS4 & 7.4 \\
\hline TLN2 & 2.7 \\
\hline UBE2O & 3.7 \\
\hline UBP47 & 5.1 \\
\hline & \\
\hline
\end{tabular}

$\mathbf{G}$

\begin{tabular}{|c|}
\hline $\mathbf{5}$ hits with \\
putative LIR \\
"RNA related \\
proteins"
\end{tabular}

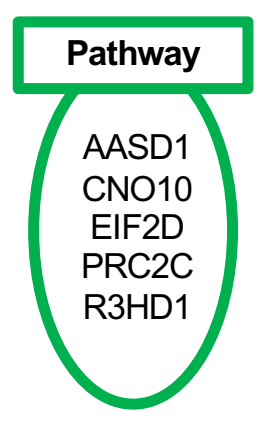

B

29 hits with putative LIR "endocytosis / membrane trafficking / endosome"

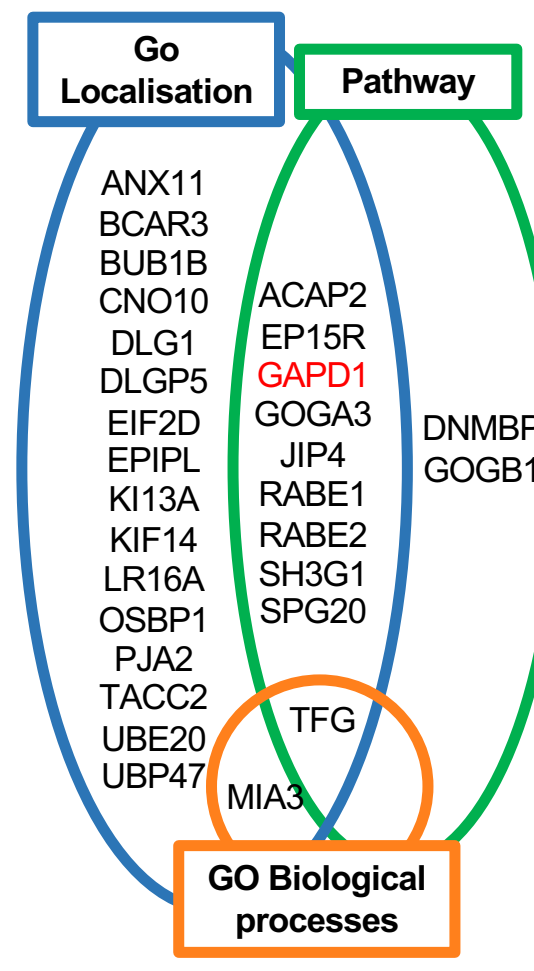

$\mathbf{F}$

$\mathbf{E}$

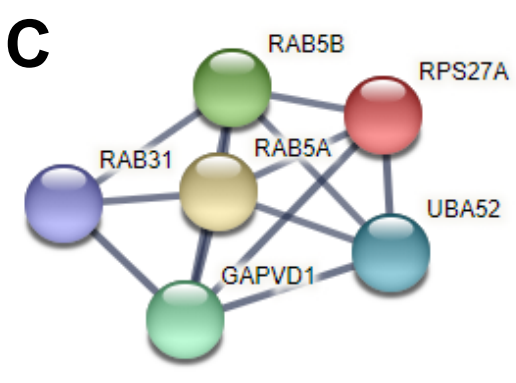

D

HeLa HeLa APEX2-GFPGFP- LC3B LC3B
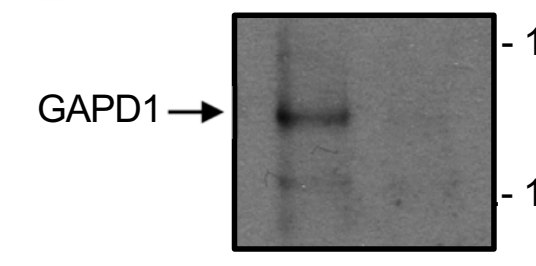

190

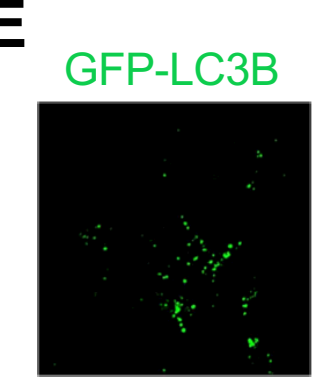

HeLa GFP-LC3B
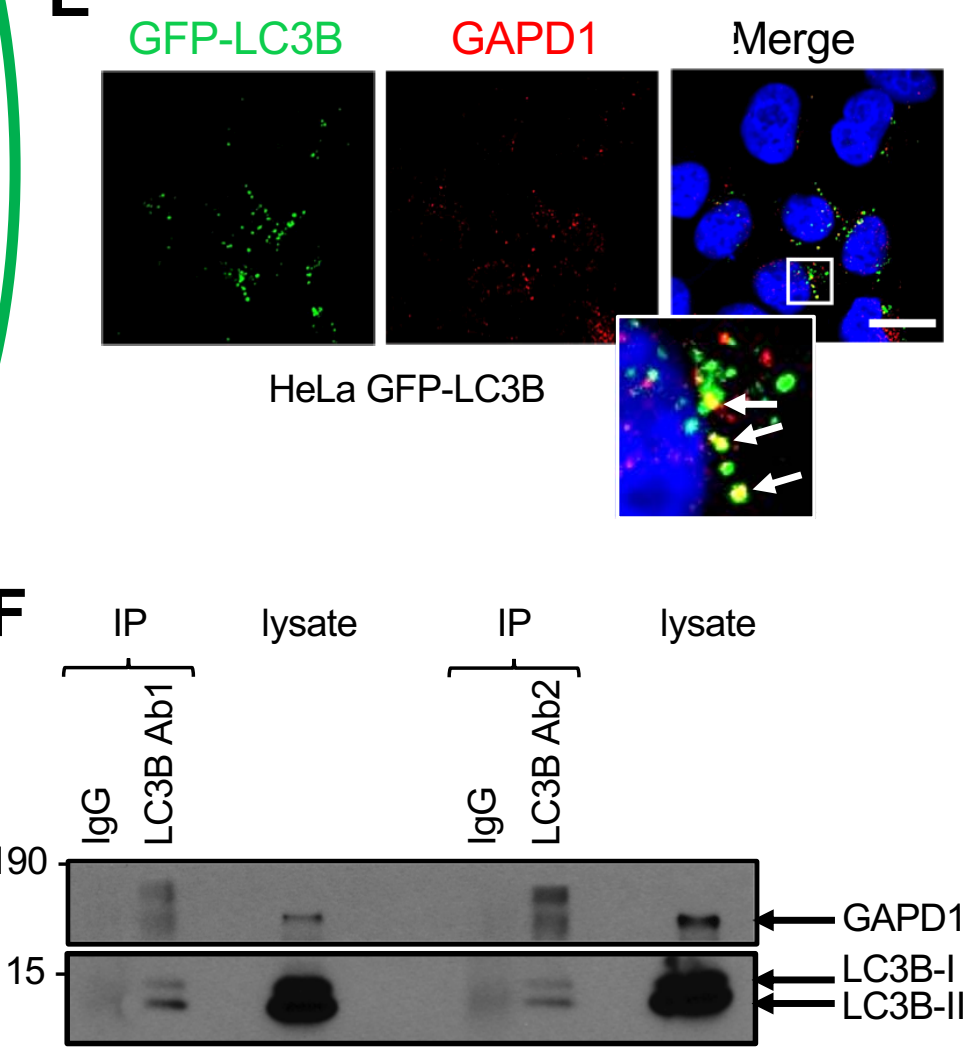

HeLa
H
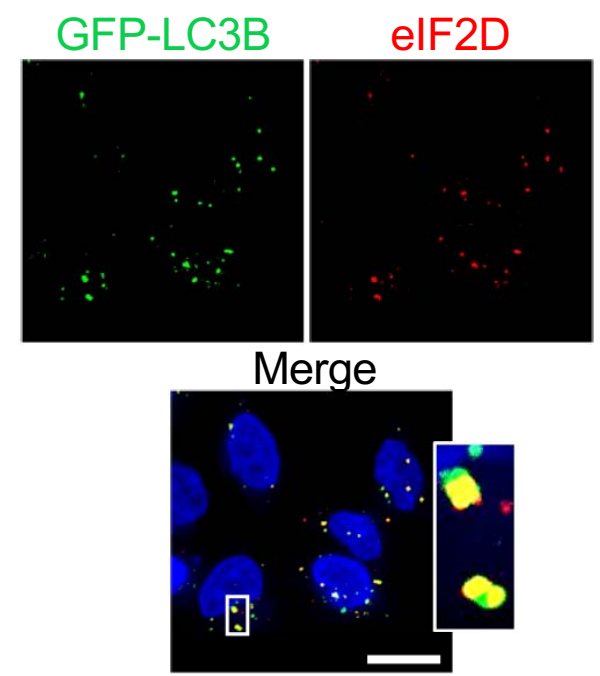

HeLa GFP-LC3B
I
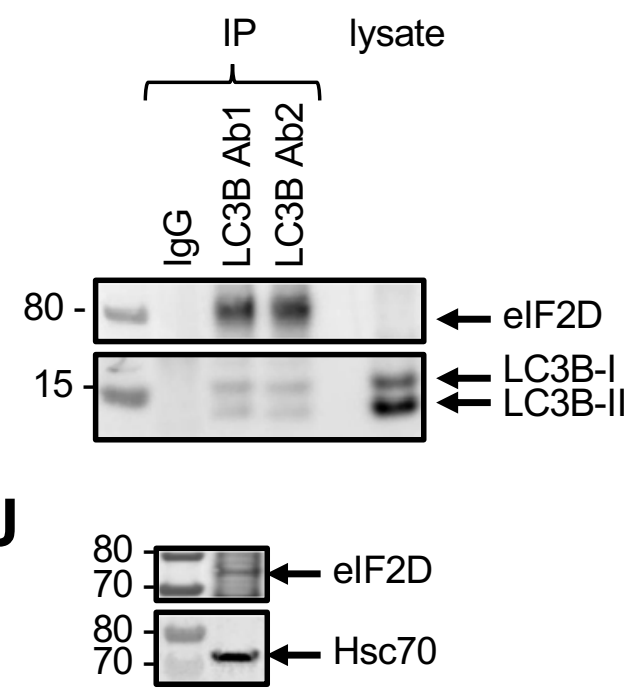